\title{
Nucleation of Small Silicon Carbide Dust Clusters in AGB Stars
}

\author{
David Gobrecht ${ }^{1,4}$, Sergio Cristallo ${ }^{1}$, Luciano Piersanti ${ }^{1}$, and Stefan T. Bromley ${ }^{2,3}$ \\ ${ }^{1}$ Osservatorio Astronomico di Teramo, INAF, I-64100 Teramo, Italy \\ ${ }^{2}$ Departament de Cincia de Materials i Química Fisica and Institut de Química Terica i Computacional (IQTCUB),Universitat de Barcelona, \\ E-08028 Barcelona, Spain \\ ${ }_{4}^{3}$ Institucio Catalana de Recerca i Estudis Avancats (ICREA), E-08010 Barcelona, Spain \\ ${ }^{4}$ Instituut voor Sterrenkunde, Celestijnenlaan 200 D, B-3001 Heverlee (Leuven), Belgium \\ Received 2017 February 2; revised 2017 March 30; accepted 2017 April 12; published 2017 May 15
}

\begin{abstract}
Silicon carbide ( $\mathrm{SiC})$ grains are a major dust component in carbon-rich asymptotic giant branch stars. However, the formation pathways of these grains are not fully understood. We calculate ground states and energetically low-lying structures of $(\mathrm{SiC})_{n}, n=1,16$ clusters by means of simulated annealing and Monte Carlo simulations of seed structures and subsequent quantum-mechanical calculations on the density functional level of theory. We derive the infrared (IR) spectra of these clusters and compare the IR signatures to observational and laboratory data. According to energetic considerations, we evaluate the viability of $\mathrm{SiC}$ cluster growth at several densities and temperatures, characterizing various locations and evolutionary states in circumstellar envelopes. We discover new, energetically low-lying structures for $\mathrm{Si}_{4} \mathrm{C}_{4}, \mathrm{Si}_{5} \mathrm{C}_{5}, \mathrm{Si}_{15} \mathrm{C}_{15}$, and $\mathrm{Si}_{16} \mathrm{C}_{16}$ and new ground states for $\mathrm{Si}_{10} \mathrm{C}_{10}$ and $\mathrm{Si}_{15} \mathrm{C}_{15}$. The clusters with carbon-segregated substructures tend to be more stable by $4-9 \mathrm{eV}$ than their bulk-like isomers with alternating $\mathrm{Si}-\mathrm{C}$ bonds. However, we find ground states with cage geometries resembling buckminsterfullerens ("bucky-like") for Si12C12 and Si16C16 and low-lying stable cage structures for $n \geqslant 12$. The latter findings thus indicate a regime of cluster sizes that differ from small clusters as well as from large-scale crystals. Thus-and owing to their stability and geometry - the latter clusters may mark a transition from a quantum-confined cluster regime to a crystalline, solid bulk-material. The calculated vibrational IR spectra of the ground-state $\mathrm{SiC}$ clusters show significant emission. They include the $10-13 \mu \mathrm{m}$ wavelength range and the $11.3 \mu \mathrm{m}$ feature inferred from laboratory measurements and observations, respectively, although the overall intensities are rather low.
\end{abstract}

Key words: dust, extinction - ISM: molecules - stars: AGB and post-AGB - stars: atmospheres

\section{Introduction}

Dust is ubiquitous in the universe and plays a crucial role in astrophysical environments. Dust impacts the synthesis of complex organic molecules in molecular clouds, the wind-driving of evolved stars, and the formation of celestial bodies (e.g., asteroids, planets) in protoplanetary discs (Dorfi \& Hoefner 1991; Ehrenfreund \& Charnley 2000; Testi et al. 2014). Dust is thus essential for the chemical evolution of galaxies, and its formation in late-type stars is the subject of this paper. The dust formation from a gaseous medium requires several thermodynamic conditions: densities above a certain threshold to ensure sufficient collisions between the constituent particles, moderate temperatures below the stability threshold of the dust component, and sufficient time for the nucleation and growth of molecular clusters into larger grains. Such conditions are found in the warm and dense molecular layers in the circumstellar environments of asymptotic giant branch (AGB) stars (Woitke et al. 1999). It is thus not surprising that among the stellar sources of dust, AGB stars are a significant contributor. We note, however, that the bulk of dust present in the local universe could arguably be the result of grain growth and reprocessing in the interstellar medium (Draine et al. 2009).

The amount and nature of the dust depends on stellar mass, metallicity, and not least on the photospheric $\mathrm{C} / \mathrm{O}$ ratio. For $\mathrm{C} / \mathrm{O}<1$ (M-type AGB stars) the circumstellar chemistry is oxygen-dominated, and the type of dust that is forming is made of silicates, alumina, and other metal oxides (Gobrecht et al. 2016). In $\mathrm{C}$-rich stars with $\mathrm{C} / \mathrm{O}>1$, carbonaceous molecules prevail and condensates such as amorphous carbon and silicon carbide $(\mathrm{SiC})$ constitute the dust grains (Henning 2010). About $90 \%$ of SiC grains are thought to come from low-mass AGB stars of approximately solar metallicity (Davis 2011) and $\mathrm{SiC}$ accounts for about $\sim 10 \%$ of carbonaceous dust of solar and moderately subsolar metallicity (Zhukovska \& Henning 2013).

S-type AGB stars are reckoned as transitional objects between M-type and C-type stars, respectively, and have little excess of either $\mathrm{C}$ or oxygen $(\mathrm{O})$. However, these stars may produce dust in the form of pure metals (Ferrarotti \& Gail 2006).

Often it is argued that dust formation and the related mass loss phenomena are less understood in M-type stars, owing to the low opacity of O-rich condensates in the near-infrared (IR) range (Woitke 2006; Höfner 2008). The wind-driving in C-rich AGB atmospheres is better understood. However, the synthesis of carbonaceous dust clusters and the formation routes toward (silicon) carbon grains is not yet fully understood.

One of the major dust components is $\mathrm{SiC}$ that shows a spectral emission/absorption in the $10-13 \mu \mathrm{m}$ range, in particular a strong and characteristic feature around $\sim 11.3 \mu \mathrm{m}$ (Speck et al. 1996, 2006). Laboratory studies have shown that the spectral band profile depends on the size, shape, and purity, respectively, of the $\mathrm{SiC}$ dust grains but is less affected by its crystal type (Mutschke et al. 1999). Fundamental lattice vibrations (i.e., phonons) dominate the interaction of IR radiation with crystalline $\mathrm{SiC}$. For small dust clusters, however, the situation is different. Owing to the lack of periodicity and lattices it is impossible to excite collective lattice vibrations such as phonons in small clusters. However, clusters possess distinct and non-bulk-like vibrational and rotational modes arising due to the bending and stretching of internal bonds.

Moreover, $\mathrm{SiC}$ dust grains have been found in primitive meteorites (Bernatowicz et al. 1987) and have typical sizes of 
0.3-3 $\mu \mathrm{m}$ (Amari et al. 1994). The analysis of the SiC grain isotope composition, in particular the excess of ${ }^{13} \mathrm{C}$ and ${ }^{15} \mathrm{~N}$ compared with scaled solar abundances, reveals unambiguously that $\mathrm{SiC}$ grains originate from the extended atmospheres of AGB stars (Hoppe et al. 1996). In addition, the majority of the $\mathrm{SiC}$ grains exhibit $s$-process isotopic signatures arising in the atmospheres of C-rich AGB stars (Gallino et al. 1994; Liu et al. 2014, 2015). More recently, new instruments like the NanoSIMS (Zinner et al. 2007) have become available and allow the analysis of $\mathrm{SiC}$ grains with sizes as small as a few nm (Hynes \& Gyngard 2009). The investigation and analysis of rather small $\mathrm{SiC}$ grains $(0.2-0.5 \mu \mathrm{m})$ have revealed that submicrometer-sized grains originating from AGB stars are much more abundant than their larger, micron-sized counterparts (Hoppe et al. 2010; Amari et al. 2014).

The classification of individual $\mathrm{SiC}$ grains into different groups named mainstream, $\mathrm{AB}$, and $\mathrm{X}$ grains, respectively, is based on the isotopic excess. Mainstream grains are associated with C-rich AGB stars. About $90 \%$ of the presolar SiC grains are thus thought to come from low-mass AGB stars of approximately solar metallicity (Davis 2011).

Owing to the interaction with the stellar radiation field, $\mathrm{SiC}$ grains are promising candidates to trigger mass-loss in C-rich AGB stars. However, the formation of $\mathrm{SiC}$ grains in stellar winds remains poorly understood.

In the bulk phase, $\mathrm{SiC}$ exists in about 250 crystalline forms, called polytypes. The most commonly encountered polytypes are $\alpha-\mathrm{SiC}$ and $\beta$-SiC with tetrahedrally coordinated $\mathrm{Si}$ atoms. All $\mathrm{SiC}$ grains extracted from meteorites have proven to be either cubic $\beta(3 \mathrm{C})-\mathrm{SiC}(\sim 80 \%)$ or hexagonal $\alpha(2 \mathrm{H})-\mathrm{SiC}$ (Daulton et al. 2003; Bernatowicz et al. 2005). These two polytypes do not differ systematically in their spectral signatures (Mutschke et al. 1999). The band profile is rather affected by grain size, shape, and impurities, respectively. Moreover, the analyzed SiC grains do not contain any seed nuclei of a different chemical type in their centers (Stroud \& Bernatowicz 2005), thus indicating a homogeneous (homomolecular) grain formation. However, the properties of nanoparticles with sizes below $50 \mathrm{~nm}$ differ significantly from bulk properties. Quantum and surface effects of these small particles lead to noncrystalline structures whose characteristics (geometry, coordination, density, binding energy) may differ by orders of magnitude as compared to the bulk material. In the smallest clusters, namely dimers and polymers of a dust species, the interatomic bonds are often unsaturated (in terms of atomic coordination), owing to the high surface-to-volume ratio. A top-down approach, i.e., deducing cluster characteristics from bulk material properties, is thus inappropriate. Instead a bottom-up approach, starting with prevalent molecules in the gas phase (e.g., $\mathrm{SiC}, \mathrm{SiO}$ ) and successive growth to clusters by molecular (addition) reactions, seems to be suitable. Such a method has been applied for clusters of magnesium oxide (Koehler et al. 1997), titanium dioxide (Lee et al. 2015), silicates of enstatite and forsterite stoichiometry (Goumans \& Bromley 2012), and silicon oxide (Bromley et al. 2016), respectively.

In Section 2 we describe the computational methods used to characterize the $\mathrm{SiC}$ cluster structures and energetics. Section 3 gathers and summarizes the results for the most stable clusters. Finally, we discuss the results with particular attention to the implications for circumstellar dust formation and spectroscopic signatures.

\section{Methods}

In this study, global optimization techniques and molecular dynamic (MD) simulations are used to determine the energetically most stable cluster structures. The more atoms a cluster contains, the larger its size is and the number of possible structural isomers increases drastically. The investigation of large clusters is therefore computationally demanding. In order to reduce the computational effort, we apply several preselection methods to find the potential minimum-energy $\mathrm{SiC}$ cluster structures. Seed cluster structures are constructed by hand according to their geometries reported in the literature.

\subsection{Monte Carlo Basin-Hopping Search on the Buckingham Potential Energy Landscape}

Some of the candidate structures are found with the Monte Carlo-Basin Hopping (MC-BH) global optimization technique (Wales \& Doye 1997) with interatomic Buckingham pair potentials. The general form of the interatomic Buckingham pair potential reads:

$$
U\left(r_{i j}\right)=\frac{q_{i} q_{j}}{r}+A \exp \left(-\frac{r_{i j}}{B}\right)-\frac{C}{r_{i j}^{6}},
$$

where $r_{i j}$ is the relative distance of two atoms, $q_{i}$ and $q_{j}$ are the charges of atom $i$ and $j$ respectively, and $A, B$, and $C$ are the Buckingham pair parameters. The first term represents the Coulomb law; the second term denotes the short-range, steric repulsion term accounting for the Pauli principle; and the last term describes the van der Waals interaction. The steric repulsion term is motivated by the fact that atoms are not dotlike but occupy a certain volume in space.

In the case of $\mathrm{SiC}$, parameter sets for the $\mathrm{Si}-\mathrm{C}$ system are lacking in the literature for several reasons. As an integral part, the electrostatic Coulomb potential appears in Equation (1). It describes the repulsion and attraction of charged particles, in this case the $\mathrm{Si}$ and $\mathrm{C}$ ions within an $\mathrm{SiC}$ cluster. As the lightest Group IV elements in the periodic table, $\mathrm{Si}$ and $\mathrm{C}$ form strong covalent bonds. The electronegativity $(\mathrm{EN})$ of carbon (EN $(\mathrm{C})=2.55)$ is too small to allow carbon to form $\mathrm{C}^{4-}$ or $\mathrm{C}^{4+}$ ions. The Buckingham potential is thus mainly used for materials with an ionic character and as, e.g., metal oxides. Nevertheless, there is a significant amount of charge transfer of 2.5 electrons between $\mathrm{Si}$ and C atoms (Watkins et al. 2009). Nonetheless, Watkins et al. (2009) have shown the similarity of zincblende $\mathrm{ZnO}$ (a cubic crystal type with face-centered lattice points) and $\beta \mathrm{SiC}$, despite the first being generally regarded as an ionic II-VI system and the latter as a covalent IV-IV system. Moreover, they found that the Buckingham parameters for $\mathrm{ZnO}$ also describe $\mathrm{SiC}$ clusters fairly well. We therefore performed $\mathrm{MC}-\mathrm{BH}$ with a simplified version of the parameter set for $\mathrm{ZnO}$ given by Whitmore et al. (2002).

The $\mathrm{ZnO}$ forcefield we employ has been shown to be able to stabilize a wide range of different cluster isomers (Al-Sunaidi et al. 2008) and bulk polymorphs (Demiroglu et al. 2014) that exhibit alternating cation-anion ordering. But to reduce the probability of missing stable cluster isomers in our searches, we also ran some test calculations for several sizes with a forcefield parameterized for $\mathrm{ZnS}$ (Wright \& Jackson 1995), which potentially provides an additional source of cluster isomers not easily found with the $\mathrm{ZnO}$ forcefield. However, the few structures that we found exclusively with the $\mathrm{ZnS}$ parameters 
had high energies (when converted to SiC clusters) and did not compete with the $\mathrm{ZnO}$ cluster analogues. Although the use of forcefields is an approximation, their use enables us to perform tractable thorough searches. With our mixed-forcefield approach (see also Section 2.2), we hope to have minimized the probability of missing a stable $\mathrm{SiC}$ isomer.

\subsection{Tersoff Potential Simulated Annealing}

As already explained, the Buckingham pair potential may fail to describe stable cluster configurations, which show the segregation of the $\mathrm{Si}$ and $\mathrm{C}$ atoms. In this case, the stable clusters are characterized by covalent rather than ionic bonds. A simple two-body interaction is thus not sufficient to properly describe the Si-C system. In addition, a three-body potential is needed to describe the covalent character of bond bending and stretching (Stillinger \& Weber 1985; Vashishta et al. 2007). In order to properly describe the internal interactions of the most stable $\mathrm{SiC}$ clusters, empirical bond-order potentials are favorable, in particular for small clusters (Erhart \& Albe 2005). This class of interatomic potentials includes the Tersoff type (Tersoff 1989), the Brenner (Brenner 1990), and the ReaxFF (van Duin et al. 2001), which take into account the bonding environment -i.e., the bond length, the angle, and the number of bonds. As a consequence of geometry, the bonding angle in a tetrahedrally coordinated system like $\mathrm{SiC}$ is $\Theta=\arccos (-1 / 3)=109.47^{\circ}$. The general form of a bond-order potential reads as follows:

$$
V\left(r_{i j}\right)=f_{c}\left(r_{i j}\right)\left[V_{\text {rep }}\left(r_{i j}\right)+b_{i j} V_{\text {att }}\left(r_{i j}\right)\right],
$$

where $V_{\text {rep }}\left(r_{i j}\right)=A_{i j} \exp -\lambda_{i j} r_{i j}$ is the repulsive part of the potential and $V_{\text {att }}\left(r_{i j}\right)=B_{i j} \exp -\mu_{i j} r_{i j}$ is the attractive effective potential. $b_{i j}$ modifies the strength of the bond, depending on the environmental parameter like the bonding angle $\Theta$ as reported in Tersoff (1989). In the Tersoff parametrization of the interatomic $\mathrm{Si}-\mathrm{C}$ molecular system, which is chosen in our approach, the potential is modified by a taper function $\mathrm{f}_{c}$. $\mathrm{f}_{c}$ is 1 for interatomic distances of $r_{i j}$ smaller than or equal to typical bonding distances and falls quickly to zero for distances larger than $S$ and thus restricts the interaction to the first neighboring atoms within a distance $S$.

$$
f_{c}\left(r_{i j}\right)= \begin{cases}1, & r_{i j}<R \\ 0.5+0.5 \cos \left(\frac{\pi\left(r_{i j}-R\right)}{S-R}\right), & R<r_{i j}<S . \\ 0, & r_{i j}>S\end{cases}
$$

The parameter set given by Tersoff (1989) suffers from an underestimation of the dimer binding energy and may not be satisfactory for the description of small gas-phase molecules and clusters. A revised set of parameters is available (Erhart \& Albe 2005). In the updated parameter set, the bond-order term $b_{i j}$ is formulated differently from the original description. Unfortunately, the new formulation is not compatible for calculations in most MD programs. However, the classic Tersoff parametrization is sufficient for our purposes, as the results are subsequently refined using a quantum mechanical level of theory. We use the General Utility Lattice Programme (GULP) (Gale 1997), which is tailored for the classic parametrization by Tersoff (1989).

Some $\mathrm{SiC}$ cluster structures have been reported in the literature (Pradhan \& Ray 2004; Hou \& Song 2008; Duan et al. 2013). We tested their stability against (small) distortions in MD runs with GULP. Furthermore, we applied the classic Tersoff potential to these structures. In the majority of cases, this potential sufficed to stabilize the structures. In some cases, however, the Tersoff potential failed to stabilize the clusters and hand-constructed structures were taken instead for the subsequent computation. In some of these failure cases, new unreported clusters appeared.

We also performed simulated annealing (SA) runs using the Tersoff-optimized structures - an imitation of a cluster cooling process. The melting point, where crystalline $\mathrm{SiC}$ decomposes, is around $3000 \mathrm{~K}$, which was chosen as the maximal temperature in the annealing routine. By varying the starting temperature $T_{\max }$ and the cooling timescales, we performed several hundred SA runs for the previously defined seed cluster structures. All the structures were cooled to a final temperature of $200 \mathrm{~K}$. We distinguished between four regimes:

1. High-temperature annealing with $T_{\max }=3000 \mathrm{~K}$.

2. Moderate-temperature annealing with $T_{\max }=1800 \mathrm{~K}$.

3. Low-temperature annealing with $T_{\max }=1000 \mathrm{~K}$.

4. $\mathrm{MD}$ at a constant temperature of $300 \mathrm{~K}$.

The majority of the investigated clusters already stabilize at around $600 \mathrm{~K}-800 \mathrm{~K}$. In order to reinforce the convergence of the MD runs, the structures were optimized to the Tersoff potential at every step where the temperature was decreased. In the MD runs at a constant temperature, snapshots of the lowest potential energy configurations were selected and further inspected.

\subsection{Quantum-mechanical Refinement}

Once preoptimized, the clusters are refined using quantummechanical density functional theory (DFT) calculations to obtain structure-specific IR spectra (i.e., vibrational frequencies), rotational constants, and zero-point-energies. By comparing the obtained IR spectra with observational data, the specific isomers present in circumstellar envelopes can thus be identified. The $(\mathrm{SiC})_{n}$ cluster structures so far reported in the literature rely on various theoretical quantum chemistry methods. They include DFT methods using generalized gradient approximation (GGA, PBE); local density approximation (LDA); B3LYP and M11 functionals, respectively; and post-Hartree-Fock methods using Møller-Plesset (MP2, MBPT) and coupled-cluster (LCCD, CCSD) techniques. For DFT methods the computational cost scales with the system size as between the order $\mathcal{O}\left(N^{3}\right)$ and $\mathcal{O}\left(N^{4}\right)$, where $N$ is the number of electrons in the cluster. This means that these methods can be readily applied to systems containing tens of atoms. However, many DFT methods can suffer from artificial electron self-interaction that results in an overly strong electron delocalization and potential energies that are too low. In contrast, post-Hartree-Fock methods do not suffer from these effects. However, the computational cost of these latter methods is very high and scales with the system size as $\mathcal{O}\left(N^{5}\right)-\mathcal{O}\left(N^{7}\right)$. They are thus prohibitive for systems of more than approximately 10 atoms. Functionals such as B3LYP and M11 attempt to compensate for the above-mentioned shortcomings of typical GGA/LDA functionals. The recent extensive benchmark study by Byrd et al. (2016) confirms that the M11 functional can correctly identify all investigated $(\mathrm{SiC})_{n}$ ground states. Although B3LYP was found to be less accurate than M11 for SiC clusters, we also include data calculated with this widely used functional for comparison. We conclude that 
for our purposes the M11 functional method is the best compromise between a reasonable computational cost and the required accuracy.

Owing to their high computational costs, DFT calculations are performed on supercomputers using the well-parallelized code Gaussian 09. These calculations approximate the wave functions and the energy of a quantum many-body system in a stationary state. In the case of SiC clusters, the hybrid B3LYP functional with a correlation-consistent polarized Valence Triple Zeta basis set is used (Becke 1993). However, recent investigations revealed that the B3LYP functional may fail to predict the correct ground states and spacings in relative energies for SiC clusters (Byrd et al. 2016). Moreover, the authors indicated in their benchmark study that the Minnesota functionals (e.g., M11, Peverati \& Truhlar 2012) have a more adequate accuracy, compared with B3LYP. For this reason we additionally performed DFT calculations using the M11 functional for the majority of the investigated clusters.

Gaussian 09 optimizes cluster structures at standard conditions (i.e., a pressure of 1 atm and a temperature of $298 \mathrm{~K}$ ). In circumstellar envelopes, however, very different conditions prevail: pressures are four to nine orders of magnitude lower and temperatures are higher by three to two hundred orders of magnitude higher. In order to account for the above-mentioned temperatures and pressures, the thermodynamic potential functions (enthalpy, entropy, Gibbs energy) are evaluated with the help of partition functions. These functions and their derivatives are calculated from the electronic energies, moments of inertia, and vibrational frequencies within the rigid-rotor harmonic oscillator approximation (McQuarrie \& Simon 1999; Goumans \& Bromley 2012).

As a consequence, the relative energy spacings of the individual clusters shift and may cross. This implies that the initial lowest-energy isomer may not be the most favorable structure in circumstellar conditions and that a different cluster structure is preferred. It is thus necessary to study a range of the energetically lowest-lying structures for each cluster size. The use of partition functions relies on the validity of thermodynamic equilibrium. We note, however, that AGB atmospheres may depart from equilibrium as they are periodically crossed by pulsational shock waves. The resulting Gibbs free energies thus have limited validity. Nonetheless, they provide a good approximation for the individual cluster stability in circumstellar conditions.

\section{Results}

In this section we describe our results on the $(\mathrm{SiC})_{n}$, $n=1-16$ clusters. We constrain our calculations to a maximum size of $n \leqslant 16$. On the one hand, the DFT calculations rapidly become more costly with increasing size. On the other hand, we follow a bottom-up approach, thus focusing on the initial steps of $\mathrm{SiC}$ dust nucleation. These steps often represent the bottleneck of cluster nucleation processes. The displayed numbers correspond to values obtained with the M11 functional, whereas the values in parentheses correspond to B3LYP results.

$\mathrm{SiC}$ : As a diatomic molecule the $\mathrm{SiC}$ monomer is a linear structure. The $\mathrm{SiC}$ triplet represents the ground state of this molecule and is $1.34(0.96) \mathrm{eV}$ lower in energy than the corresponding singlet state. We find an average bond length of 1.707 (1.813) $\AA$, a rotational constant of 20.6 (18.3) GHz, and a
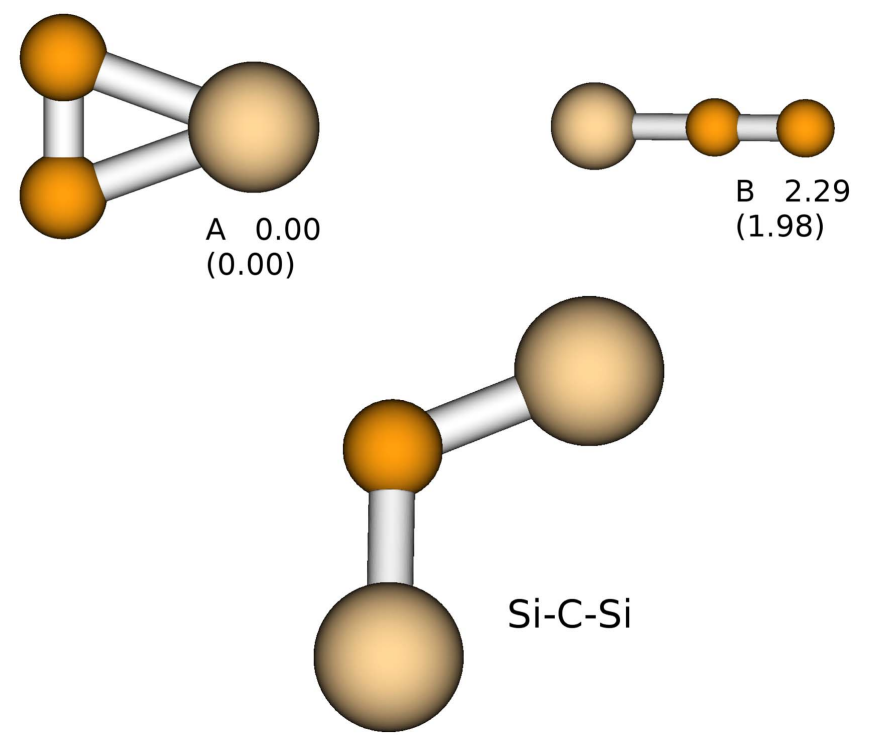

Figure 1. The two stable $\mathrm{SiC}_{2}$ clusters and the $\mathrm{Si}_{2} \mathrm{C}$ ground state with relative energies (in $\mathrm{eV}$ ).

vibrational frequency of $1008.7(862.2) \mathrm{cm}^{-1}$, which corresponds to a wavelength of 9.9 (11.6) $\mu \mathrm{m}$.

$\mathrm{Si}_{2} \mathrm{C}$ and $\mathrm{SiC}_{2}$ : The $\mathrm{SiC}_{2}$ ground state is a triangle and is lower by $2.29(1.98) \mathrm{eV}$ than the linear triplet isomers $\mathrm{B}$ in Figure 1 . The isomer with a linear $\mathrm{C}-\mathrm{Si}-\mathrm{C}$ chain is unstable $(6.82 \mathrm{eV}$ above the ground state). Regarding the large differences in energy, we assume that isomer $\mathrm{A}$ is the dominant state of $\mathrm{SiC}_{2}$ and that the geometry of $\mathrm{B}$ is negligible for all temperatures and pressures.

Reilly et al. (2015) characterized the ground electronic state of $\mathrm{Si}_{2} \mathrm{C}$. The singlet isomer with two off-axis $\mathrm{Si}$ atoms bent by an angle of $114.87 \mathrm{deg}$. and a $\mathrm{C}_{2 v}$ symmetry, reported in McCarthy et al. (2015) and Cernicharo et al. (2015), is found by our M11 calculations, but not with the B3LYP functional. In the latter case, the molecule relaxes into a linear $\mathrm{C}-\mathrm{Si}-\mathrm{C}$ chain or fails to converge. This result demonstrates the advantage of the M11 functional compared with the B3LYP functional. The linear $\mathrm{Si}-\mathrm{Si}-\mathrm{C}$ isomer exhibits imaginary frequencies in the IR spectra. Structures showing imaginary frequencies (vibration modes) represent a saddle point (and not a minimum) in the complex potential energy landscape. These saddle points have as the real minimum a zero gradient and are interpreted as transition states.

Thus, the bent $\mathrm{C}-\mathrm{Si}-\mathrm{C}$ structure is the only stable cluster we report for $\mathrm{Si}_{2} \mathrm{C}$.

$\mathrm{Si}_{2} \mathrm{C}_{2}$ : Two structures of $\mathrm{Si}_{2} \mathrm{C}_{2}$ are commonly proposed as ground states: the linear triplet structure and the closed rhomb. They usually show a tiny difference in binding energies and are thus considered as degenerate isomers. The exact energy separation depends on the used functional and basis set. This is consistent with our B3LYP findings, where these two structures are separated by only $0.03 \mathrm{eV}$, as can be seen in Figure 2. On the contrary, we show that the M11 functional predicts the rhombic structure (A) to be more stable than the linear chain (C) by $0.69 \mathrm{eV}$ at standard conditions. The isomer (B) is characterized by threefold-coordinated ( $\mathrm{Si}$ and $\mathrm{C}$ ) atoms and is $0.39(0.26) \mathrm{eV}$ above the lowest-lying state. The linear triplet structure (D) has a potential energy that is $2.77(2.20) \mathrm{eV}$ higher with respect to the ground state. The structures shown here have been previously found by Pradhan \& Ray (2004), 

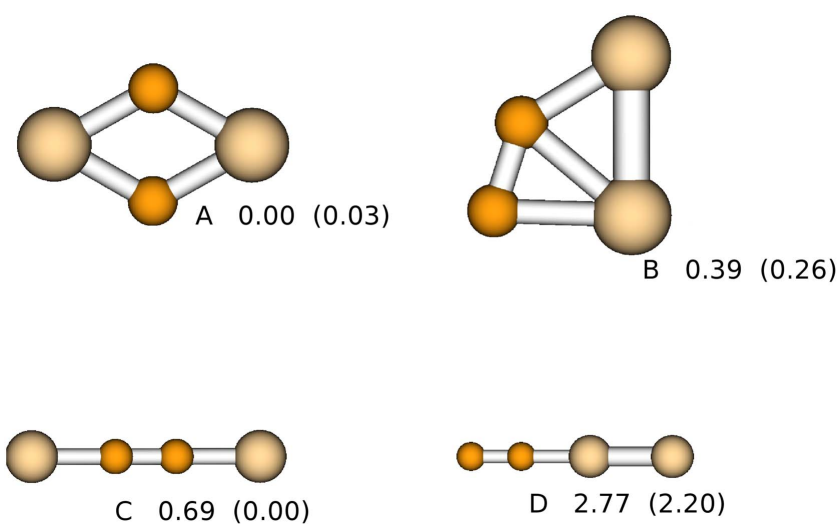

Figure 2. The most stable $\mathrm{Si}_{2} \mathrm{C}_{2}$ clusters and relative energies (in $\mathrm{eV}$ ).

Hou \& Song (2008), and Duan et al. (2010), respectively. Several further isomers have been investigated by Pradhan \& Ray (2004). However, our calculations show that the structures $\mathrm{G}, \mathrm{H}$, and $\mathrm{I}$ in Figure 3 are transition states, and the force constants indicate a relaxation into isomer $\mathrm{B}$ of Figure 2.

$\mathrm{Si}_{3} \mathrm{C}_{3}$ : For $\mathrm{Si}_{3} \mathrm{C}_{3}$ clusters an extensive and comprehensible study has been carried out by Mühlhäuser et al. (1994), who examined 17 structures in total. Further studies by Hou \& Song (2008), Pradhan \& Ray (2004), and Duan et al. (2010) have revealed that some of these structures are particularly stable. In Figure 4, we summarize our findings.

Apparently, the isomers in Figure 4 are characterized by planar structures as well as three-dimensional forms with triangular faces. All clusters contain three adjacent $\mathrm{C}$ atoms. The majority of the found $\mathrm{Si}_{3} \mathrm{C}_{3}$ clusters show a carbon chain (like $\mathrm{C}$ and $\mathrm{D}$ ), but a triangular $\mathrm{C}$ arrangement is also observed (A and B). The ground-state A and the next higher-lying isomer $\mathrm{B}$ are nonplanar and have triangular faces. Structure D is the lowest-lying isomer within the B3LYP level of theory. In the M11 functional frame, however, D is $0.88 \mathrm{eV}$ above the minimum structure and the $\mathrm{C}_{3}$ chain is slightly bent. Meanwhile, some structures that have been reported in the literature exhibit imaginary IR frequencies, indicating a transition state rather than a minimum structure. By identifying the bond causing the imaginary vibration and by reoptimizing a slightly distorted structure, we found that structures $\mathrm{M}, \mathrm{N}$, and $\mathrm{O}$ in Figure 5 relax into other low-lying structures. All linear structures are triplet states and are energetically unfavorable or exhibit imaginary frequencies and can thus not be considered as minimum structures.

$\mathrm{Si}_{4} \mathrm{C}_{4}$ : The ground state (A) of $\mathrm{Si}_{4} \mathrm{C}_{4}$ displayed in Figure 6 is a nonplanar structure with one $\mathrm{Si}$ atom out of the plane. The second lowest energy structure (B) is a planar structure with a $\mathrm{C}_{2 h}$ symmetry. It is composed of a four-member trans- $\mathrm{C}$ chain and can be viewed as two connected $\mathrm{Si}_{2} \mathrm{C}_{2}$ clusters (isomer B in Figure 2), bridged by $\mathrm{C}-\mathrm{C}$ bonding. The corresponding cisisomer (structure $\mathrm{C}$ ) has an energy of $0.33 \mathrm{eV}$ above the ground state and $0.07(0.09) \mathrm{eV}$ above the trans-isomer. Trans- and cisisomers differ only by a rotation of $180^{\circ}$ along the $\mathrm{C}-\mathrm{C}$ doublebond axis. The structures A-D have been reported in Duan et al. (2013) and references therein.

Cluster structures with alternating $\mathrm{Si}-\mathrm{C}$ bonds have been found by means of Monte Carlo simulations applying the Buckingham pair potential. These structures show a high degree of symmetry and are displayed in Figure 7. Structure M, with $a T_{d}$ symmetry reported by Watkins et al. (2009), has an
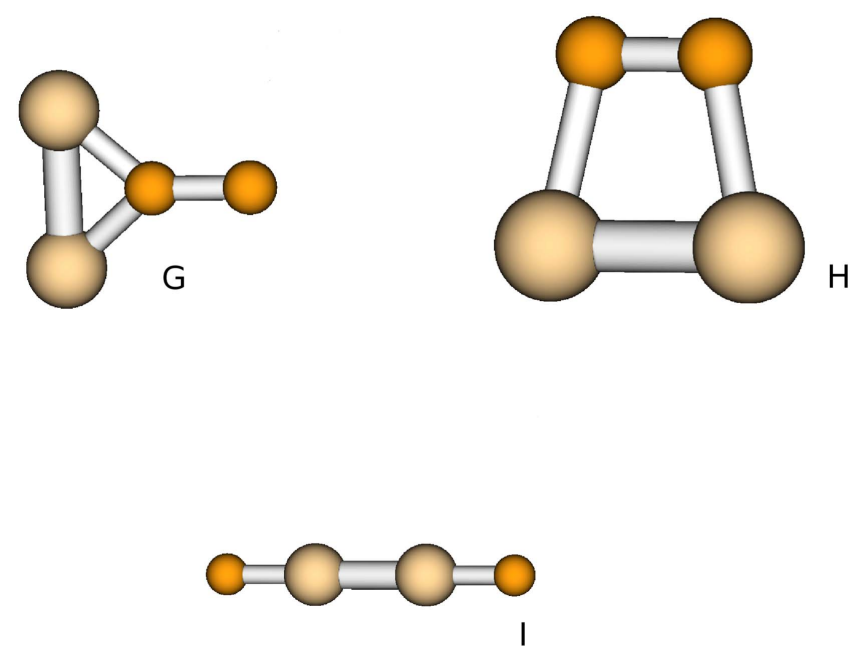

Figure 3. Transition states of $\mathrm{Si}_{2} \mathrm{C}_{2}$ clusters.
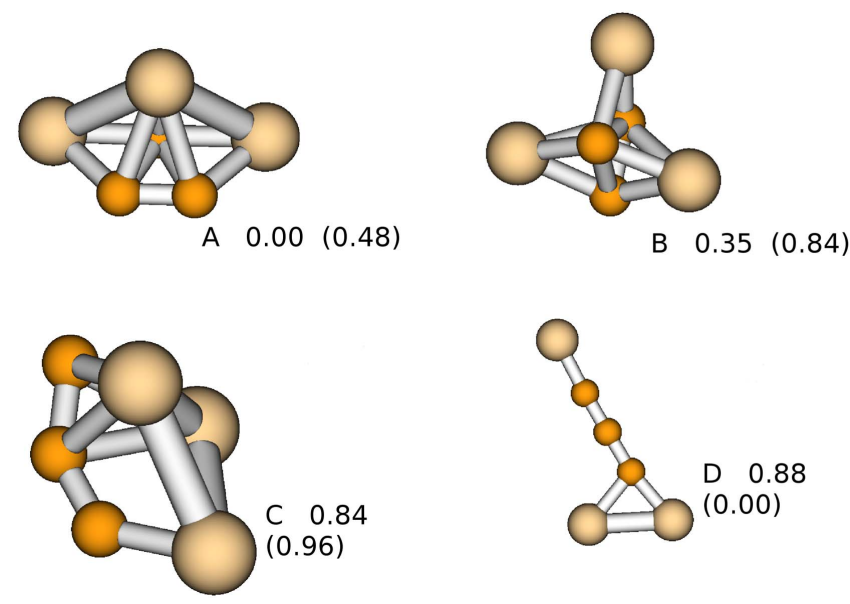

Figure 4. The most stable $\mathrm{Si}_{3} \mathrm{C}_{3}$ clusters and relative energies (in $\mathrm{eV}$ ).
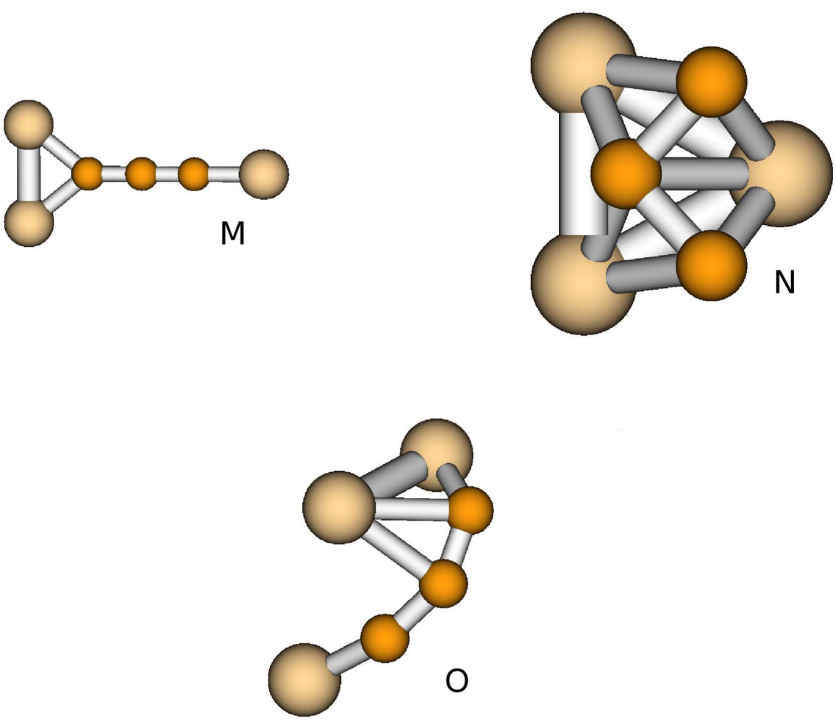

Figure 5. Transition states of $\mathrm{Si}_{3} \mathrm{C}_{3}$ clusters.

energy of $5.89 \mathrm{eV}$ above the ground state. The other isomers we found have energies $(\sim 3-7 \mathrm{eV})$ far above the ground state. Our results thus indicate that for the size of $n=4$, the MC$\mathrm{BH}$-generated $\mathrm{SiC}$ clusters cannot compete with the segregated 

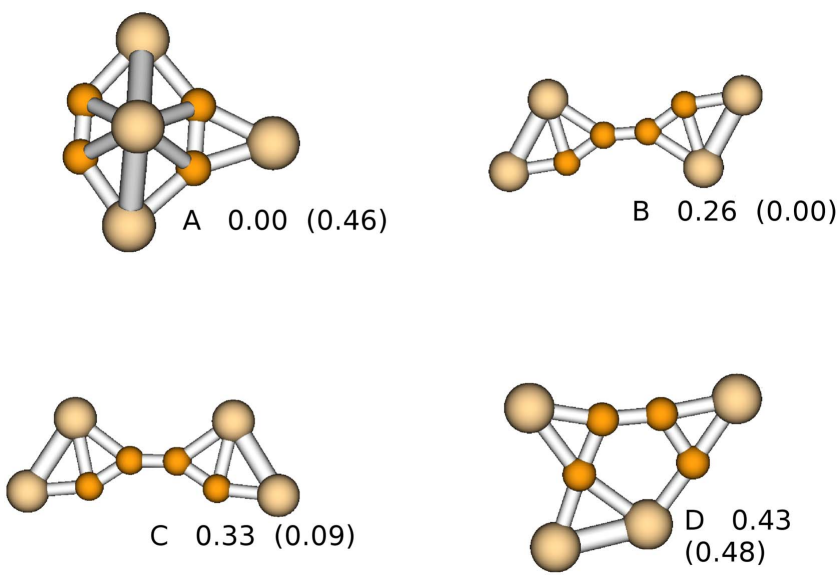

Figure 6. The most stable $\mathrm{Si}_{4} \mathrm{C}_{4}$ clusters and relative energies (in $\mathrm{eV}$ ).
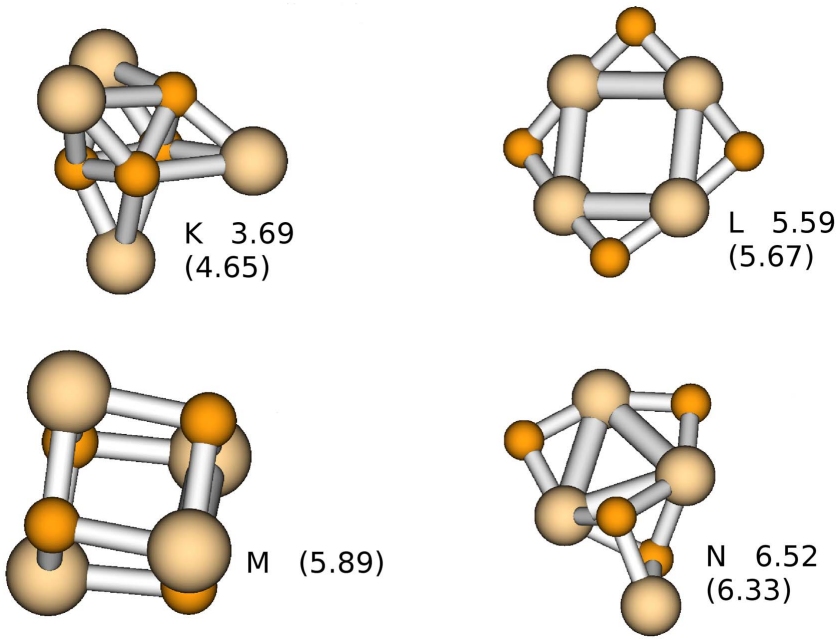

Figure 7. Symmetric $\mathrm{Si}_{4} \mathrm{C}_{4}$ clusters with alternating $\mathrm{Si}-\mathrm{C}$ bonds.

structures in Figure 6. An ab initio exploration of the potential energy surfaces of this cluster size has been performed by Bertolus et al. (2004).

$\mathrm{Si}_{5} \mathrm{C}_{5}$ : This is the smallest cluster size, where a $\mathrm{C}$ ring appears. The ground-state cluster (A) exhibits a $\mathrm{C}_{5}$ ring and a mirror plane, thus belonging to the $\mathrm{C}_{s}$ symmetry group. Structure $\mathrm{B}$ also shows a $\mathrm{C}_{s}$ symmetry and a six-member ring with five $\mathrm{C}$ atoms and one $\mathrm{Si}$ atom. Both structures (A and $\mathrm{B}$ ) have the lowest potential energy in the B3LYP calculations as well. All low-lying structures displayed in Figure 8 exhibit either a bent $\mathrm{C}$ chain with five members or a ring, and are nonplanar. These structures were previously reported by Duan et al. (2013).

$\mathrm{Si}_{6} C_{6}$ : The ground state of $\mathrm{Si}_{6} \mathrm{C}_{6}$ contains a $\mathrm{C}_{6}$ ring and laterally distributed $\mathrm{Si}$ atoms as can be seen in Figure 9 . Isomers $\mathrm{B}$ and $\mathrm{C}$ show five-member $\mathrm{C}$ rings. Structure $\mathrm{D}$ has the lowest potential in the B3LYP frame and is a planar configuration containing two five-member rings consisting of four $\mathrm{C}$ atoms and one $\mathrm{Si}$ atom. Among the lowest-lying isomers, compound $\mathrm{A}$ is the only one found with an aromatic $\mathrm{C}_{6}$ ring. The other aromatic isomers have significantly higher potential energies. We found that structures $\mathrm{B}$ and $\mathrm{C}$ exhibit a $\mathrm{C}_{5}$ ring and a one-sided $\mathrm{Si}$ segregation (apart from single $\mathrm{Si}$ atoms conjugating the cluster). Clusters obeying a strict alternation of $\mathrm{Si}$ and $\mathrm{C}$ atoms are $5-9 \mathrm{eV}$ higher in energy
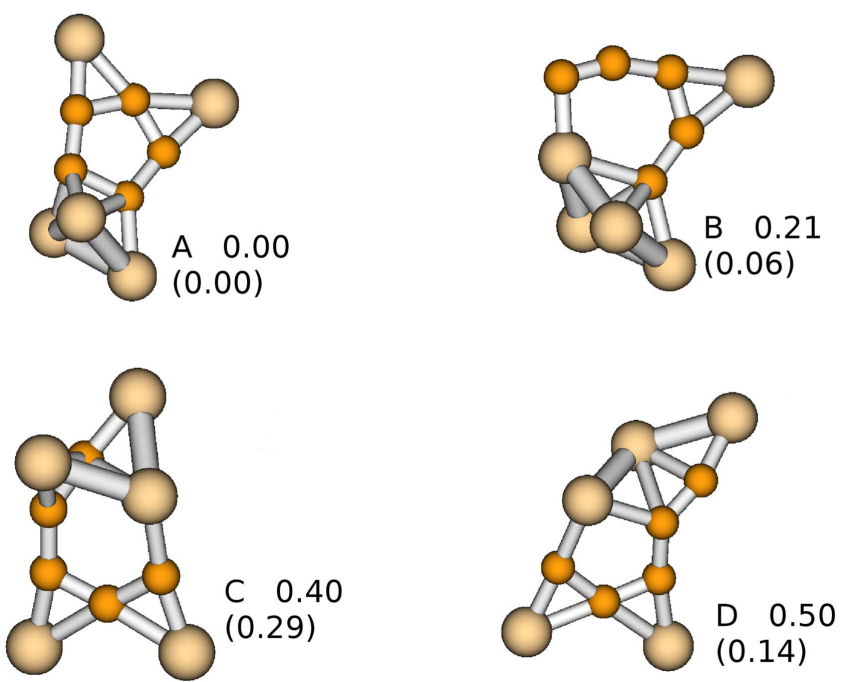

Figure 8. The most stable $\mathrm{Si}_{5} \mathrm{C}_{5}$ clusters and relative energies (in $\mathrm{eV}$ ).
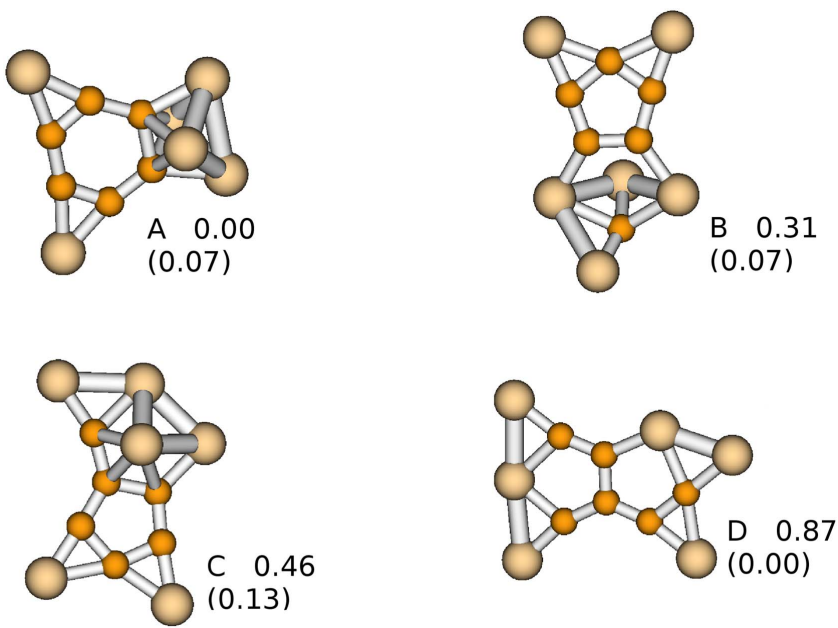

Figure 9. The most stable $\mathrm{Si}_{6} \mathrm{C}_{6}$ clusters and relative energies (in eV).
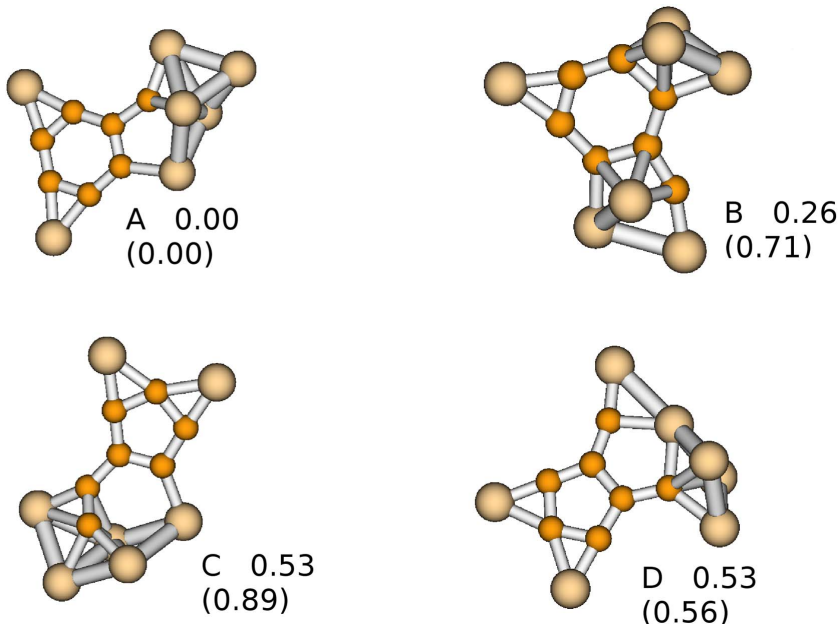

Figure 10. The most stable $\mathrm{Si}_{7} \mathrm{C}_{7}$ clusters and relative energies (in eV).

compared with the ground state. Structures A-D were reported in Duan et al. (2013).

$\mathrm{Si}_{7} \mathrm{C}_{7}$ : The ground state of $\mathrm{Si}_{7} \mathrm{C}_{7}$ (cluster A in Figure 10) consists of an aromatic ring connected to a $\mathrm{Si}_{5}$ subcluster and 

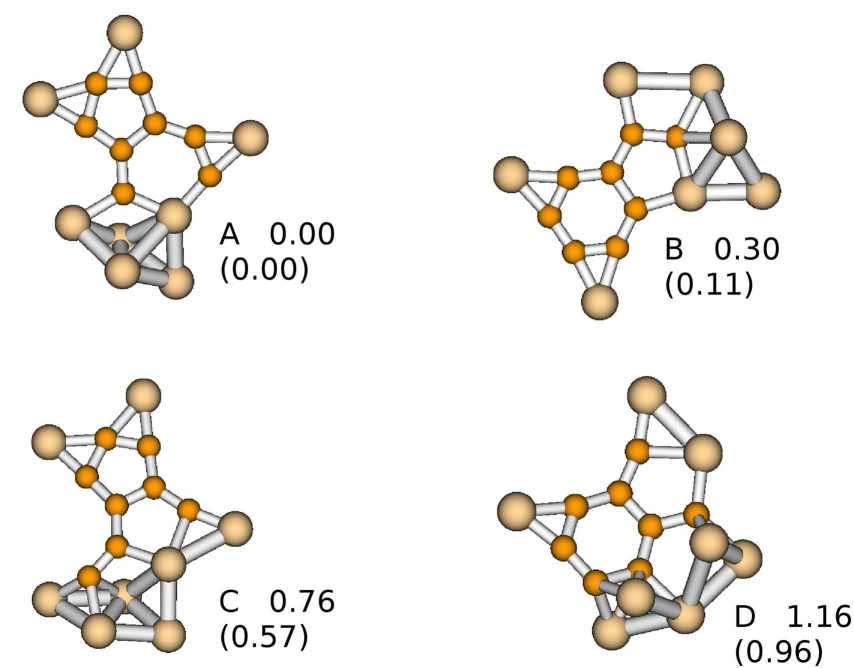

Figure 11. The most stable $\mathrm{Si}_{8} \mathrm{C}_{8}$ clusters and relative energies (in $\mathrm{eV}$ ).

two individual $\mathrm{Si}$ atoms. Structures $\mathrm{C}$ and $\mathrm{D}$ have a fivemember carbon ring in common, where the dangling bonds are saturated by two individual $\mathrm{Si}$ atoms. $\mathrm{MC}-\mathrm{BH}$-generated structures with alternating $\mathrm{Si}$ and $\mathrm{C}$ atoms have energies 7-8 eV higher than that in isomer $\mathrm{A}$.

$\mathrm{Si}_{8} \mathrm{C}_{8}$ : In the most stable $\mathrm{Si}_{8} \mathrm{C}_{8}$ clusters, the $\mathrm{C}$ atoms tend to form planar five- or six-member rings as can be seen in Figure 11. The $\mathrm{Si}$ atoms surround the $\mathrm{C}$ subcluster and segregate spatially. Structures $A$ and $C$ contain a $C_{5}$ ring, while structures $\mathrm{B}$ and $\mathrm{D}$ have a $\mathrm{C}_{6}$ ring. The remaining $\mathrm{C}$ atoms arrange as side-chains to form a silicon-substituted ring. Structures A, B, and C are reported in Duan et al. (2013). Other candidate isomers (except structure D) obtained through simulated annealing (SA) are $3-5 \mathrm{eV}$ above the minimum structure. The highly symmetric double-ring structure $(\mathrm{G})$ proposed by Belenkov et al. (2012) and the "keyhole" isomer $(\mathrm{H})$ in Figure 12 have potential energies of 5.29 (6.04) eV and $4.46(5.35) \mathrm{eV}$ above the ground state, respectively. Further structures with alternating arrangements of atom types ( $\mathrm{Si}$ and C) have energies of 4-9 eV above the ground state.

$\mathrm{Si}_{9} \mathrm{C}_{9}$ : The energetically favorable structures $\mathrm{A}, \mathrm{B}$, and $\mathrm{C}$ in Figure 13 contain fused $C_{6}$ and $C_{5}$ rings. Only isomer $D$ has an exceptional character with a $\mathrm{C}_{6}$ and two side chains. A $\mathrm{C}_{2 v}$ symmetric structure with alternating $\mathrm{Si}$ and $\mathrm{C}$ atoms was obtained by the MC-BH method, but it has an energy of 7.15 (7.84) $\mathrm{eV}$ above the ground state. Other isomers obtained by SA have potential energies of $2-6 \mathrm{eV}$ above the ground state.

$S i_{10} C_{10}$ : The lowest-lying isomer using the B3LYP functional (structure $\mathrm{A}^{\prime}$ ) is reported for the first time and can be seen in Figure 14. When applying the M11 functional, Structure $A^{\prime}$ relaxes into state $\mathrm{A}$. We thus consider $\mathrm{A}$ as the true ground state. Clusters A, B, and C have been found by Duan et al. (2013). It is prominent that fused double $C_{6}$ rings of the naphthalene type form for the four favorable clusters $\mathrm{A}^{\prime}, \mathrm{A}, \mathrm{B}$, and $\mathrm{C}$. Compared to smaller $\mathrm{SiC}$ cluster sizes, the most stable $\mathrm{Si}_{10} \mathrm{C}_{10}$ clusters have spatial and open cage-like forms rather than planar configurations. We find that the 11 energetically most favorable clusters reside in a narrow energy range of $1 \mathrm{eV}$. This is more than for any other size of the investigated $\mathrm{SiC}$ clusters. Further isomers we found by means of the SA of seed clusters have energies of $2-4 \mathrm{eV}$ above the minimum energy structure. MC-BH synthesized isomers have energies of 4-6 eV above the ground state.
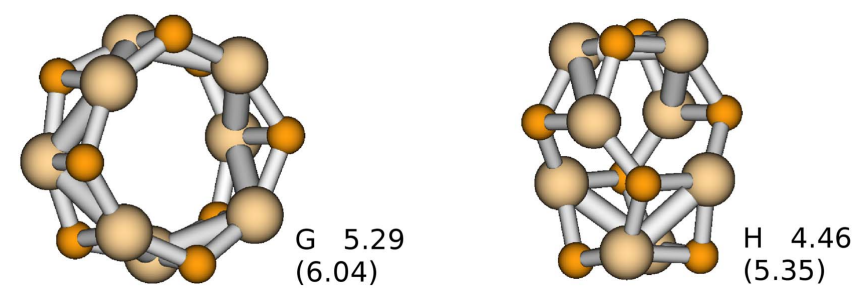

Figure 12. $\mathrm{Si}_{8} \mathrm{C}_{8}$ cluster structures with alternating $\mathrm{Si}-\mathrm{C}$ bonds. Relative energies are given in $\mathrm{eV}$.
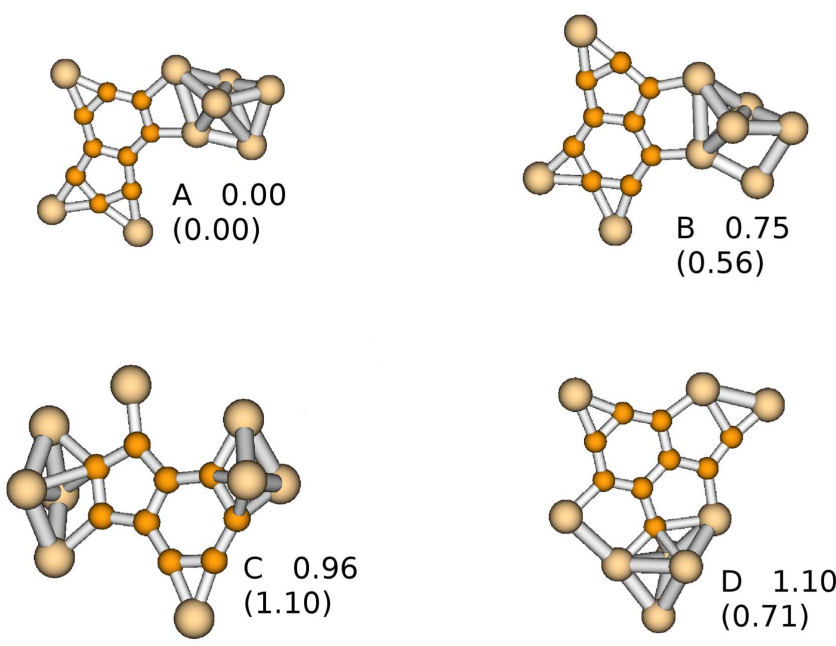

Figure 13. The most stable $\mathrm{Si}_{9} \mathrm{C}_{9}$ clusters and relative energies (in $\mathrm{eV}$ ).
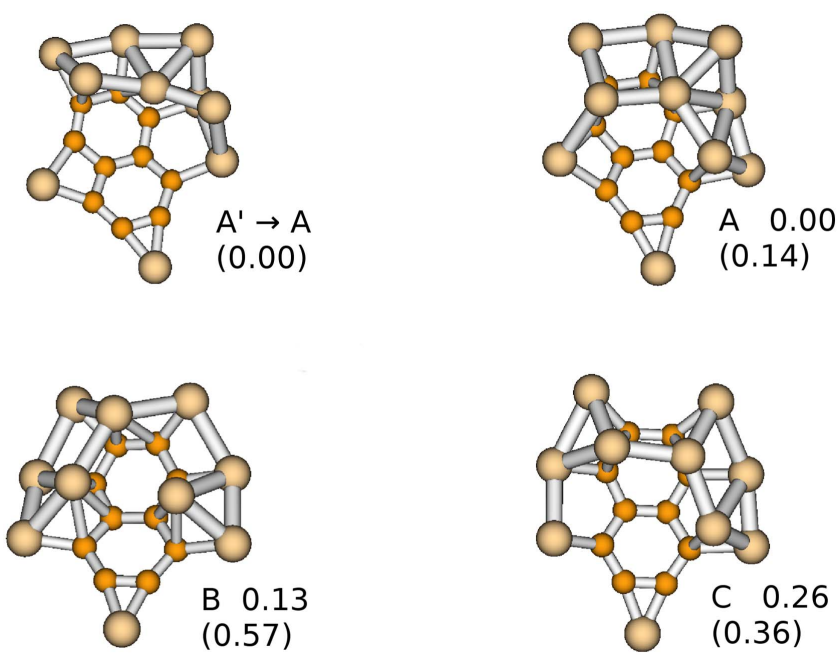

Figure 14. The most stable $\mathrm{Si}_{10} \mathrm{C}_{10}$ clusters and relative energies (in $\mathrm{eV}$ ).

$S i_{11} C_{11}$ : The most stable isomers (see Figure 15) are characterized by the presence of a $\mathrm{C}_{6}$ ring and two $\mathrm{C}_{5}$ rings, respectively, each one sharing an edge with another ring. Structures A-D are found in Duan et al. (2013). All structures, except the ground state $\mathrm{A}$, have a $\mathrm{C}$ subunit characterized by a fusion of two $\mathrm{C}_{5}$ rings and one $\mathrm{C}_{6}$ ring. The ground state $\mathrm{A}$ contains one $\mathrm{C}_{5}$ ring and one $\mathrm{C}_{6}$ ring, and two Si-substituted five-member rings $\left(\mathrm{C}_{4} \mathrm{Si}\right.$ rings $)$. For this cluster size, stable cage-like clusters found with the MC-BH method possess potential energies of $1.2-4.5 \mathrm{eV}$ above the ground state.

$S i_{12} C_{12}$ : The ground state (A) of $\mathrm{Si}_{12} \mathrm{C}_{12}$ is displayed in Figure 16 and represents a particular case in the series of $\mathrm{Si}_{n} \mathrm{C}_{n}$ ground states. It exhibits a highly symmetric tetrahedral 

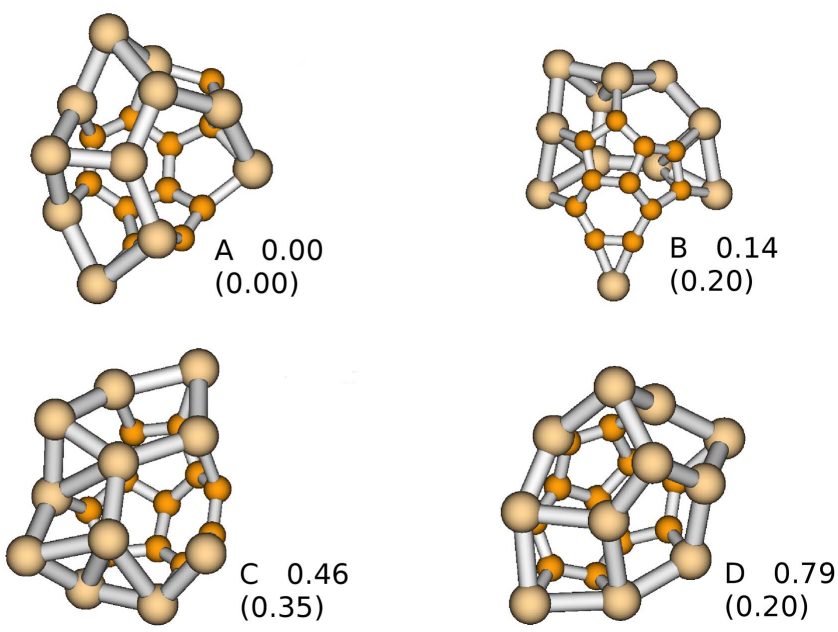

Figure 15. The most stable $\mathrm{Si}_{11} \mathrm{C}_{11}$ clusters and relative energies (in $\mathrm{eV}$ ).
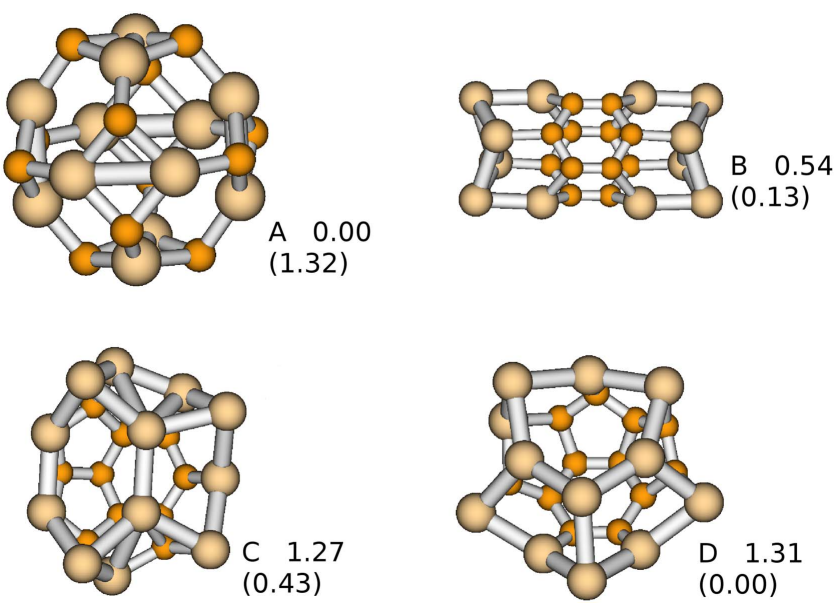

Figure 16. The most stable $\mathrm{Si}_{12} \mathrm{C}_{12}$ clusters and relative energies (in $\mathrm{eV}$ ).

configuration (symmetry group $T_{\mathrm{H}}$ ) with alternating $\mathrm{SiC}$ bondings and was proposed by Watkins et al. (2009) as a potential nano-building block of larger structures. With a "bucky"-like configuration, the almost spherical structure resembles the chemical family of fullerenes. The cluster has a mass of $\sim 480$ amu and a diameter of $\sim 5.9 \AA$. This would result in a mass density of $0.919 \mathrm{~g} \mathrm{~cm}^{-3}$, which is about $30 \%$ of $3.217 \mathrm{~g} \mathrm{~cm}^{-3}$, a reference mass density for all polytypes (Patnaik 2003). Owing to its properties, structure A may link the segregated clusters with the crystalline bulk material observed in pristine $\mathrm{SiC}$ dust grains. In contrast to other isomers, structure A may be identified spectroscopically, owing to its strong IR vibration mode intensities (see Figure 17). Moreover, it is the smallest ground state among the most stable $\mathrm{Si}_{n} \mathrm{C}_{n}$ clusters that we found with the Buckingham pair potential, applying the MC-BH method. Owing to its stability, shape, and atomic coordination, structure A may be a candidate for the basic building blocks of $\mathrm{SiC}$ dust grains and may trigger the molecular size where cluster chemistry crosses over to dust chemistry (i.e., condensation and coalescence).

The second most stable polymer (B) exhibits a dihedral $\mathrm{D}_{2 H}$ symmetric structure with two unconnected $\mathrm{C}_{6}$ rings. Despite being classified as a member of the $C_{1}$ group, cluster $D$ is almost symmetric with a quasi-mirror plane. Structures $B$ and D have been reported by Song et al. (2010). Apart from

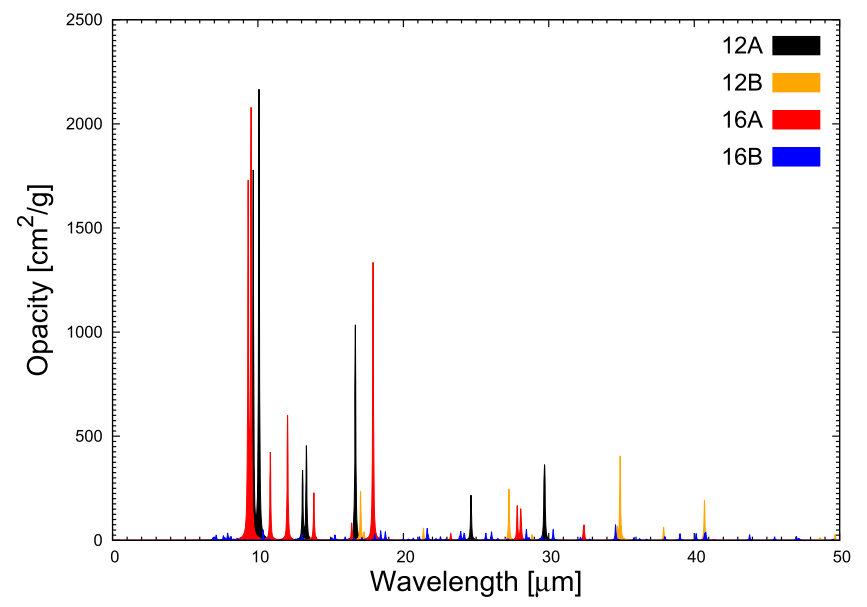

Figure 17. Vibrational IR spectra of the two lowest-lying states of Si12C12 (12A and 12B) and Si16C16 (16A and 16B). 12A and 16A correspond to the "bucky"-like configurations.

structure $\mathrm{C}$, all these clusters exhibit a fused $\mathrm{C}_{6}-\mathrm{C}_{6}-\mathrm{C}_{5}$ ring segregation. Isomer $\mathrm{C}$ has not been reported previously.

We found several other stable clusters with higher potential energies. However, they are not displayed as they have a similar open-cage-like configuration such as structure $\mathrm{C}$ and the higher-lying isomers. Among the lowest-lying $\mathrm{Si}_{12} \mathrm{C}_{12}$ configurations, a high degree of symmetry is prominent. The symmetric structures obtained with the $\mathrm{MC}-\mathrm{BH}$ method are, apart from the ground state, $8-9 \mathrm{eV}$ higher in potential energy than isomer $\mathrm{A}$.

$S i_{13} C_{13}$ : Among the lowest-lying $\mathrm{Si}_{13} \mathrm{C}_{13}$ isomers, we find $\mathrm{C}$ segregations with one $\mathrm{C}_{6}$ ring and three $\mathrm{C}_{5}$ rings (structures $A$ and $\mathrm{B}$ ) and four $\mathrm{C}_{5}$ rings (C) (see Figure 18). In addition, the $\mathrm{Si}$ atoms start to develop segregated rings with five to six members (structures A, B, and C). Structures A, B, and C have been found by Song et al. (2010), although owing to the different functional/basis set used in their study, they were found in a different energy ordering. We find that the energetic ordering is preserved by comparing our M11 and B3LYP results. Further structures found by $\mathrm{SA}$ are $0.5-4 \mathrm{eV}$ higher in potential energy. Structure $\mathrm{D}$ is an $\mathrm{MC}-\mathrm{BH}$-generated structure and contains seven 6-member rings and one large eightmember ring. We find three further structures with cage-like geometries that have potential energies of $0.56-1.93 \mathrm{eV}$ above the ground state and are thus comparable to the segregated clusters obtained by SA.

$\mathrm{Si}_{14} C_{14}$ : The two most stable isomers of $\mathrm{Si}_{14} \mathrm{C}_{14}$ both show symmetries, as can be seen in Figure 19. The first (A) shows two mirror planes and a two-fold symmetry axis $\left(\mathrm{C}_{2 \mathrm{v}}\right.$ group), while the second (B) has one mirror plane $\left(\mathrm{C}_{s}\right.$ group). The most stable structures $(\mathrm{A}, \mathrm{B}$, and $\mathrm{D})$ show a complete $\mathrm{C}$ segregation consisting of two $\mathrm{C}_{6}$ rings and two $\mathrm{C}_{5}$ rings. Whereas in structures $\mathrm{B}$ and $\mathrm{D}$ the $\mathrm{C}_{6}$ rings are connected and share a $\mathrm{C}-\mathrm{C}$ bonding, in structure $A$ the $\mathrm{C}_{5}$ rings share binding electrons and the $\mathrm{C}_{6}$ rings are separated from each other. Moreover, structures $\mathrm{B}$ and $\mathrm{C}$ show an overall open cage geometry, whereas structure A represents a closed hollow ellipsoid. Isomers A, B, and C were found in Song et al. (2010), while isomer D is reported for the first time. Further structures obtained by SA are not displayed here and have energies of $1-4 \mathrm{eV}$ above the ground state. With the $\mathrm{MC}-\mathrm{BH}$ approach we found a structure with alternating $\mathrm{Si}-\mathrm{C}$ bonds and an energy of 

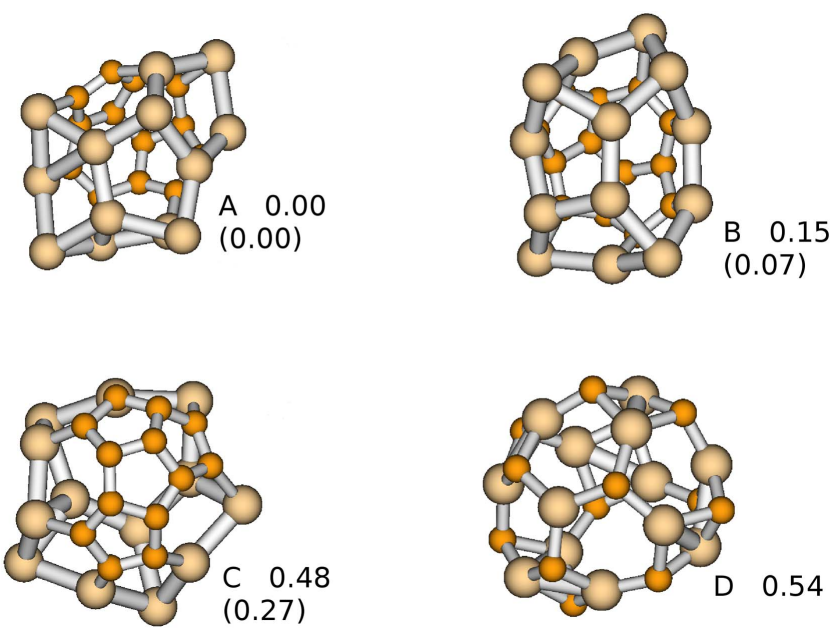

Figure 18. The most stable $\mathrm{Si}_{13} \mathrm{C}_{13}$ clusters and relative energies (in eV).

$0.64 \mathrm{eV}$ above the ground state. It is the fifth-lowest energy structure for $\mathrm{Si}_{14} \mathrm{C}_{14}$. Other stuctures obtained with the MC-BH method have energies of $1.4-1.7 \mathrm{eV}$ above the global minimum. The latter findings indicate that for $n=14$, the segregated forms cease to dominate, and cage-like clusters can compete against the latter in terms of potential energy.

$S i_{15} C_{15}$ : The ground state of $\mathrm{Si}_{15} \mathrm{C}_{15}$ is a symmetric structure with alternative bond $\mathrm{Si}-\mathrm{C}$ bonds found with the $\mathrm{MC}-\mathrm{BH}$ method. It is composed of eleven 6-member and four 4-member rings obeying a strict alternation of $\mathrm{Si}$ and $\mathrm{C}$ atoms. This ground state is reported for the first time. Its low potential energy indicates that the symmetric cage-like configurations with alternating $\mathrm{Si}-\mathrm{C}$ bonds can compete against segregated structrures at this size regime and even represent the lowest energy structure for $n=15$.

The second lowest isomer showing two separate C segregations and a quasi-mirror plane is displayed in Figure 20. It shows a similarity with the smaller-sized $(n=12)$ structure $\mathrm{B}$ in Figure 16. The two low-lying isomers (C and D) are almost identical in terms of their B3LYP potential energy and can be termed degenerate. However, an investigation of these clusters with the M11 functional reveals that they have a larger spacing in energy and that they can be regarded as independent and discrete clusters. Moreover, they show distinct geometries, vibrational IR spectra, and rotational constants. Their carbon subunit is almost identical and resembles structure D in Figure 6 of Song et al. (2010). However, the silicon atoms are arranged differently, giving rise to the change in potential energy.

Structure B has been reported previously by Song et al. (2010).

$S i_{16} C_{16}$ : The ground state of $\mathrm{Si}_{16} \mathrm{C}_{16}$ shows a particularly high degree of symmetry (point group $T_{d}$ ) and is composed of six-member rings with alternating $\mathrm{Si}-\mathrm{C}$ bondings. This structure has been put forward as the building units of larger $\mathrm{SiC}$ frameworks by Watkins et al. (2009). The overall structure is a closed hollow fullerene-like cage and shows strong IR features (around 9.3-9.5 and $18.9 \mu \mathrm{m}$; see Figure 17), compared to isomer B (and the other isomers of this size). As for $n=12$, we found the ground state structure by applying the Buckingham pair potential using MC-BH. The "bucky"like structure has an approximately spherical shape and exhibits alternating $\mathrm{Si}-\mathrm{C}$ bonds. The hollow spheres with a $T_{d}$ symmetry have a mass of $\sim 640 \mathrm{amu}$ and a diameter of $\sim 6.5 \AA$. This would result in a mass density of $0.924 \mathrm{~g} \mathrm{~cm}^{-3}$,
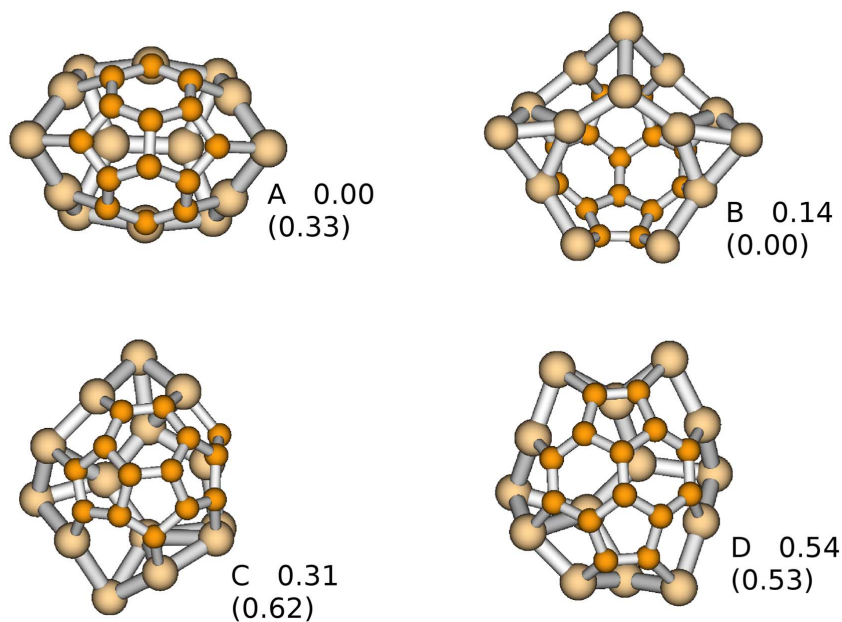

Figure 19. The most stable $\mathrm{Si}_{14} \mathrm{C}_{14}$ clusters and relative energies (in $\mathrm{eV}$ ).
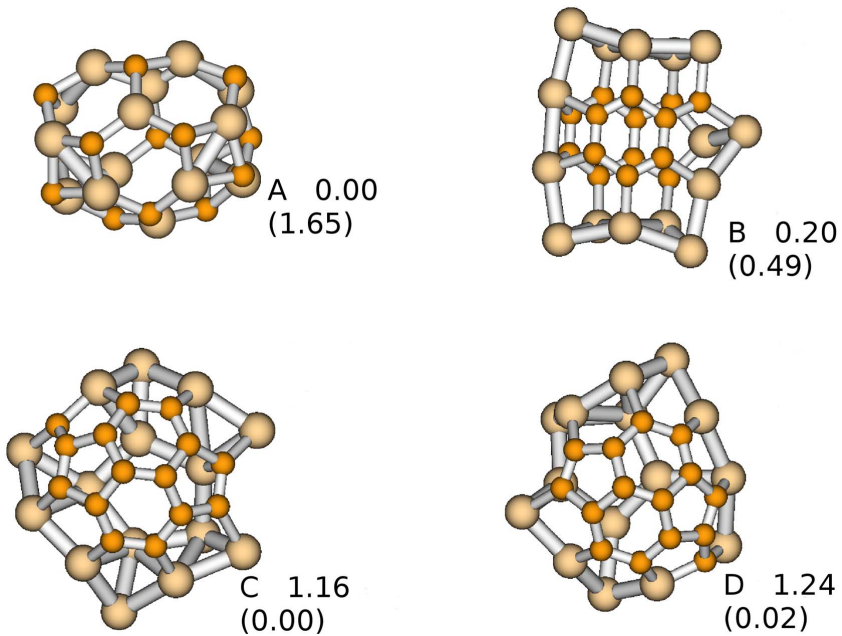

Figure 20. The most stable $\mathrm{Si}_{15} \mathrm{C}_{15}$ clusters and relative energies (in $\mathrm{eV}$ ).

which is very similar to the $n=12$ case and is about $30 \%$ of $3.217 \mathrm{~g} \mathrm{~cm}^{-3}$, a reference mass density for all polytypes (Patnaik 2003). Owing to its strong and characteristic IR features, this particular isomer can be spectroscopically identified. As for $n=12$, the ground state (A) may link the segregation-dominated small clusters $(n<12)$ with larger clusters and crystalline $\mathrm{SiC}$ bulk material.

The next higher lying isomer of $\mathrm{Si}_{16} \mathrm{C}_{16}$ (structure $\mathrm{B}$ ) shows two $\mathrm{C}$ segregations, a $\mathrm{C}_{6} \mathrm{C}_{5}$-ring and a $\mathrm{C}_{6}$-ring with a one-Catom arm-and exhibits a distorted symmetry with a quasimirror plane, as can be seen in Figure 21. Isomer B has been found for the first time and is the lowest-energy structure using the B3LYP functional. Despite its low potential energy, it is challenging to observationally detect structure A, owing to low vibrational IR intensities (see Figure 17).

For sizes $n \leqslant 12$, we clearly show the emergence of a new family of stable clusters, the cage-like structures with alternating $\mathrm{Si}-\mathrm{C}$ bonds. Some properties (alternating atomic arrangement, bond lengths) of the latter strongly resemble the bulk phase of the cubic crystal type 3C-SiC, compared to the segregated clusters. However, the "bucky" clusters are void in their interior, which is not the case for any $\mathrm{SiC}$ crystal lattice. We thus expect a transition from cage- to bulk-like structures at some not-further-specified size, $n \geqslant 16$. 

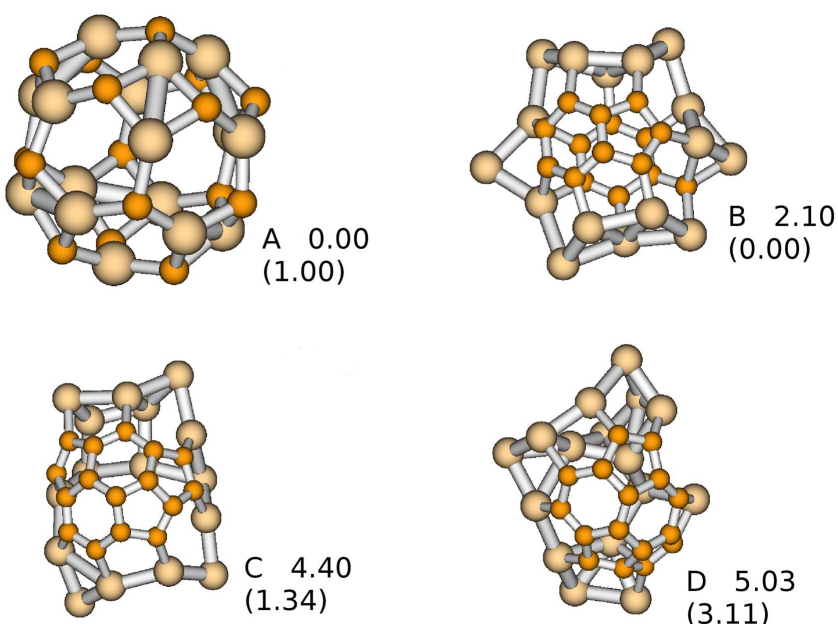

Figure 21. Stable isomers of $\mathrm{Si}_{16} \mathrm{C}_{16}$ clusters.

\subsection{Gas Conditions in the Circumstellar Envelope}

In Table 1 , the energetic feasibility for $\mathrm{SiC}$ cluster growth at characteristic circumstellar conditions is displayed.

The left upper part of Table 1 represents gas conditions shortly after the passage of a pulsational shock, where the gas is hot and compressed $(T=3000 \mathrm{~K}-5000 \mathrm{~K}, \quad p=$ $100-500$ dyne $\left.\mathrm{cm}^{-2}=10-50 \mathrm{P}\right)$. In this case, the SiC dimerization, representing the initial process of starting particle growth, is suppressed by an energy barrier of several eV.

In addition, for the larger clusters $(n \geqslant 3)$, the Gibbs free energy of formation $(\Delta G)$ of the lowest-lying clusters becomes largely positive and nucleation is unlikely to occur, owing to the lack of stability and high activation barriers.

The left intermediate part of Table $1(T=2000 \mathrm{~K}-2500 \mathrm{~K}$, $p=10-100$ dyne $\left.\mathrm{cm}^{-2}=1-10 \mathrm{P}\right)$ reflects typical conditions at the visual photosphere where the optical depth $\tau$ is $2 / 3$. Under such conditions, the initial steps for $\mathrm{SiC}$ cluster growth are likely to occur, as they proceed under the excess of energy. At some point of the nucleation chain, however, owing to energy barriers, this growth may not proceed (we refer to this as a waiting point) until the conditions in the wind have relaxed to lower temperatures and densities, where subsequent nucleation is favorable. Examples for waiting points are $\mathrm{Si}_{2} \mathrm{C}_{2}$ and $\mathrm{Si}_{3} \mathrm{C}_{3}$ and also $\mathrm{Si}_{9} \mathrm{C}_{9}$ at high temperatures, as can be seen in Figure 22.

Cooler and more diluted gas conditions prevail further away from the star $\left(\sim 10 R_{*}\right)$, where the pulsational shocks have strongly weakened and damped and the wind has accelerated up to its terminal velocity (and it is assumed that a considerable amount of dust has already formed). Such conditions $\left(T=500 \mathrm{~K}, 10^{-5}\right.$ dyne $\left.\mathrm{cm}^{-2}=10^{-6} \mathrm{~Pa}\right)$ are found in the right lower part of the table. In this regime, the complete nucleation pathway is energetically favorable. However, the densities are so low that particle collision events with subsequent nucleation become rare. Nevertheless, previously synthesized dust clusters may stochastically coalesce and form dust grains.

In summary, $\mathrm{SiC}$ cluster formation and growth favor dense and cool conditions; vice versa, the $\mathrm{SiC}$ cluster synthesis is hampered in hot and dilute environments. As circumstellar envelopes cover a broad range of temperatures and pressures in space (due to the radial distance from the star) as well as in time (owing to dynamical pulsations and wind acceleration), a
Table 1

Overview of the SiC Nucleation by Monomer Addition at Different Temperatures $T$ (in $\mathrm{K}$ ) and Pressures $p$ (in dyne $\mathrm{cm}^{-2}$ )

\begin{tabular}{ccccccc}
\hline \hline$T / p$ & 500 & 100 & 10 & 1 & 0.1 & 0.01 \\
\hline 5000 & $\times$ & $\times$ & $\times$ & $\times$ & $\times$ & $\times$ \\
3000 & $\times$ & $\times$ & $\times$ & $\times$ & $\times$ & $\times$ \\
2500 & $\mathrm{Si}_{3} \mathrm{C}_{3}$ & $\mathrm{Si}_{3} \mathrm{C}_{3}$ & $\mathrm{Si}_{2} \mathrm{C}_{2}$ & $\times$ & $\times$ & $\times$ \\
2000 & $\sqrt{ }$ & $\sqrt{ }$ & $\mathrm{Si}_{3} \mathrm{C}_{3}$ & $\mathrm{Si}_{2} \mathrm{C}_{2}$ & $\mathrm{Si}_{2} \mathrm{C}_{2}$ & $\mathrm{Si}_{2} \mathrm{C}_{2}$ \\
1500 & $\sqrt{ }$ & $\sqrt{ }$ & $\sqrt{ }$ & $\sqrt{ }$ & $\sqrt{ }$ & $\sqrt{ }$ \\
1000 & $\sqrt{ }$ & $\sqrt{ }$ & $\sqrt{ }$ & $\sqrt{ }$ & $\sqrt{ }$ & $\sqrt{ }$ \\
500 & $\sqrt{ }$ & $\sqrt{ }$ & $\sqrt{ }$ & $\sqrt{ }$ & $\sqrt{ }$ & $\sqrt{ }$ \\
\hline
\end{tabular}

Note. An energetically feasible nucleation is marked with $\sqrt{ }$. Suppressed nucleation with large energy barriers is marked with $\times$. If the nucleation pathway is partially favorable, the largest preferential cluster is given.

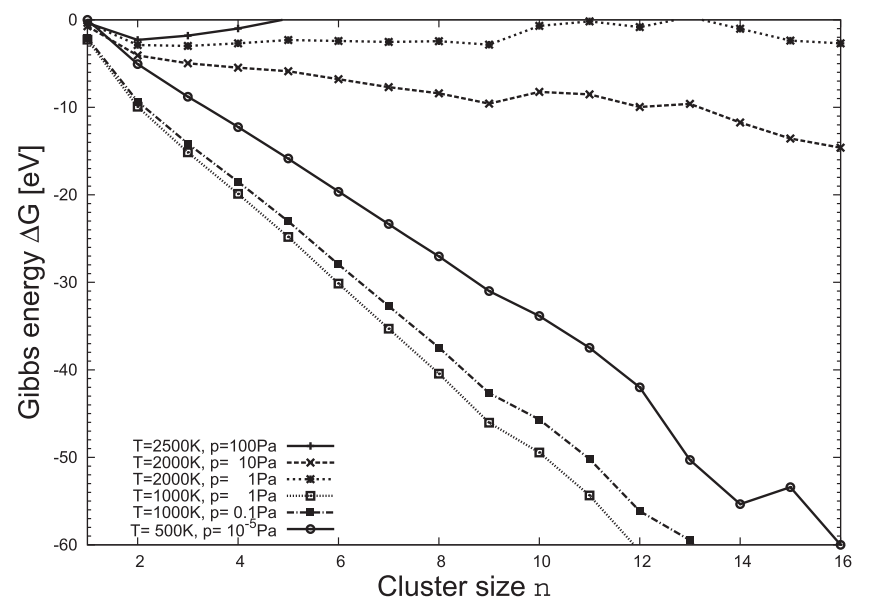

Figure 22. Gibbs energy of formation of the ground state clusters vs. cluster size for different sets of gas temperatures and pressures.

combination and exposure of various pressures and temperatures involving waiting points are more realistic than assuming thermodynamic equilibrium. Yasuda \& Kozasa (2012) showed in their calculations that $\mathrm{SiC}$ grains hardly form in local thermodynamic equilibrium and that nonequilibrium processes (like pulsations) are necessary to explain the observed ratio of $\mathrm{SiC}$ dust $(0.01-0.3)$ in carbonaceous dust grains inferred from the radiative transfer model.

For a constant pressure of $p=100 \mathrm{dyne} \mathrm{cm}^{-2}$ (which corresponds to a gas density of $3.6 \times 10^{14} \mathrm{~cm}^{-3}$ ) and $T=2500 \mathrm{~K}$, the cluster growth is energetically feasible up to $n=3$, or $\mathrm{Si}_{3} \mathrm{C}_{3}$. The synthesis of larger cluster sizes is strongly hampered by energy barriers of the order of $100 \mathrm{~kJ} \mathrm{~mol}^{-1}$ $(\sim 1 \mathrm{eV})$. At $T \leqslant 2000 \mathrm{~K}$ and reasonably high densities, the processes increasing the cluster size are energetically downhill up to the maximum size $n=16$ considered in this study.

In Figure 23, the relative binding energy of the lowest-lying $\mathrm{Si}_{n} \mathrm{C}_{n}$ cluster (ground state) is plotted versus cluster size $n$ according to the following prescription:

$$
\Delta E_{b}\left(\mathrm{Si}_{n} \mathrm{C}_{n}\right)=\frac{E_{b}\left(\mathrm{Si}_{n} \mathrm{C}_{n}\right)}{n}-E_{b}(\mathrm{SiC}) .
$$

The largest incremental in the binding energy $(4.3 \mathrm{eV})$ between clusters of size $n$ and $(n+1)$ occurs between the SiC monomer and the dimer. For larger cluster sizes the binding energy increases almost monotonically and saturates around $6.0 \mathrm{eV}$. However, we also note that the ground states of $n=9$ and $n=12$ are particularly stable. $\Delta E_{\mathrm{MO}}$ denotes the energy 


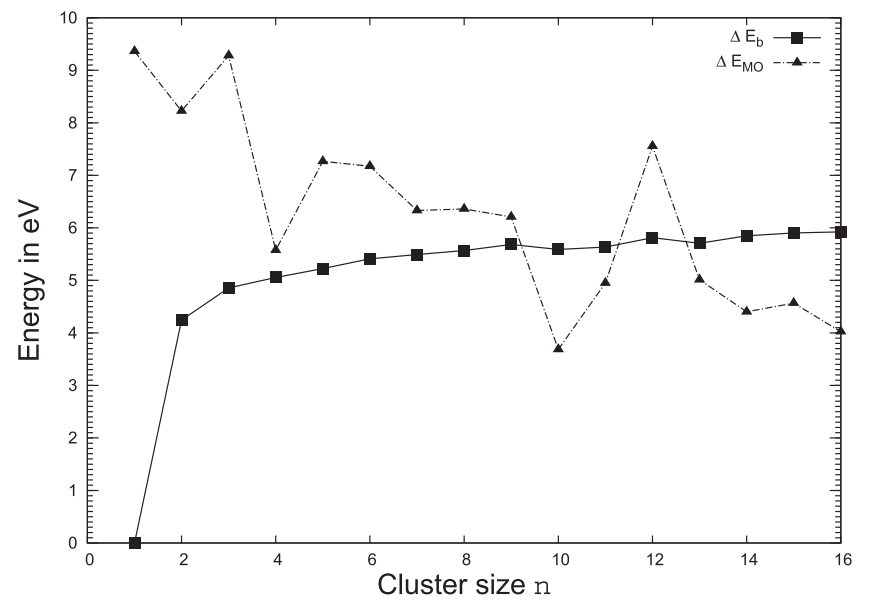

Figure 23. The relative binding energy $\Delta E_{b}$ (filled squares and solid line) of the ground state $\mathrm{Si}_{n} \mathrm{C}_{n}$ clusters (normalized to cluster size $n$ ) and the HOMOLUMO energy gap $\Delta E_{\mathrm{MO}}$ (triangles and dashed line) of the ground state $\mathrm{Si}_{n} \mathrm{C}_{n}$ clusters.

gap of the highest-occupied molecular orbital (HOMO) and the lowest-unoccupied molecular orbital (LUMO). This quantity describes the strength and stability of a given electronic configuration. A large $\Delta E_{\mathrm{MO}}$ indicates a high cluster stability against thermal and radiative excitations. Evidently, for $\Delta E_{\mathrm{MO}}$, there is no correlation with cluster size $n$. However, it reveals that some cluster sizes $(n=3,12,16)$ have a higher stability and that the closed cage structures are particularly stable compared to other cluster sizes. Generally, $\Delta E_{\mathrm{MO}}$ tends to decrease the larger the system is, as the density of (unoccupied) states increases with cluster size $n$. For a given cluster size, the HOMO-LUMO energy gap is not necessarily the largest for the ground state.

In Figure 24, curves with a vanishing Gibbs energy of formation $\left(\Delta G_{f}=0\right)$ for small $\mathrm{Si}_{n} \mathrm{C}_{n}, n \leqslant 7$, and $\mathrm{Si}_{2} \mathrm{C}$ and $\mathrm{SiC}_{2}$ are shown. Our results indicate that the latter $\left(\mathrm{SiC}_{2}\right)$ is the first $\mathrm{Si}-\mathrm{C}$ molecule to emerge from the hot atmosphere, as it is more stable over a broad range of gas pressures compared with the other considered compounds. The formation of $\mathrm{Si}_{2} \mathrm{C}$ and $\mathrm{Si}_{2} \mathrm{C}_{2}$ becomes exogonic $\left(\Delta G_{f}<0\right)$ at very similar pressures and temperatures. In contrast, the formation of the $\mathrm{SiC}$ molecule is more likely at lower temperatures assuming a constant pressure. For the larger $\mathrm{Si}_{n} \mathrm{C}_{n}$ clusters, the formation probability shifts successively to lower temperatures (or higher pressures). From Figure 24 we conclude that a homogeneous nucleation is viable, presuming a bottom-up approach in the formation of $\mathrm{Si}_{n} \mathrm{C}_{n}$ clusters. It should be noted that in the presence of stellar pulsations, a trajectory of a gas parcel will not be represented by a single line in the $T-p$ diagram but rather by a complex zigzag. This implies that certain clusters with a particularly high stability (i.e., waiting points) form at several times during the nucleation process and can thus be considered as candidates for a possible observational detection. In summary, we conclude that a homogeneous nucleation of $\mathrm{SiC}$ clusters is definitely viable in circumstellar environments and that owing to their thermal stability, the molecular species $\mathrm{Si}_{2} \mathrm{C}_{2}$ and $\mathrm{Si}_{2} \mathrm{C}$ may play an important role in the initial steps of $\mathrm{SiC}$ nucleation.

In Figure 25, the calculated vibrational IR spectra of the $\mathrm{Si}_{n} \mathrm{C}_{n}$ ground state clusters are displayed. A cluster of size $n$ (i.e., the number of $\mathrm{SiC}$ units) exhibits at maximum $6 \cdot(n-1)$ individual vibrations accounting for bending and stretching modes.

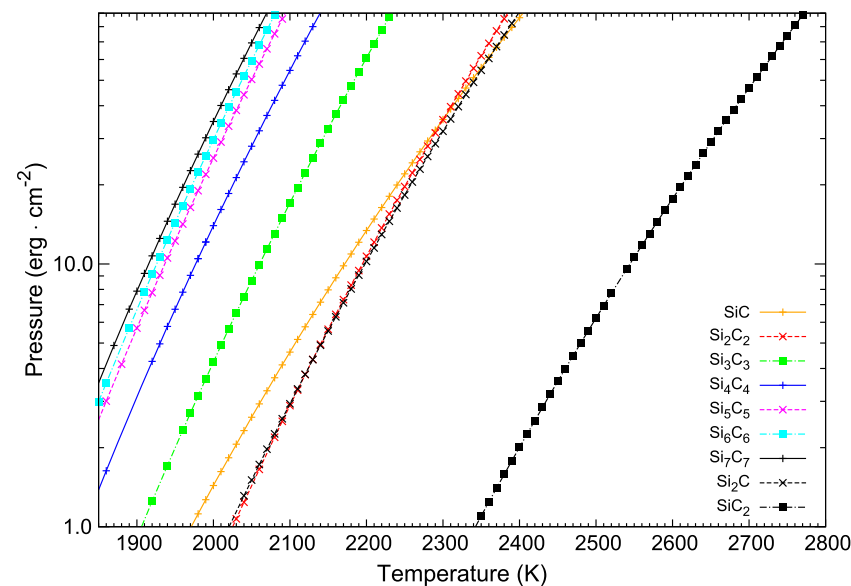

Figure 24. Curves with a vanishing Gibbs energy of formation $\left(\Delta G_{f}=0\right)$ for small ground state clusters. The corresponding cluster formation is energetically favorable $\left(\Delta G_{f}<0\right)$ for temperatures/pressures left/above of these curves. In contrast, for temperatures/pressures right/below these curves, the corresponding cluster formation is unlikely $\left(\Delta G_{f}>0\right)$.
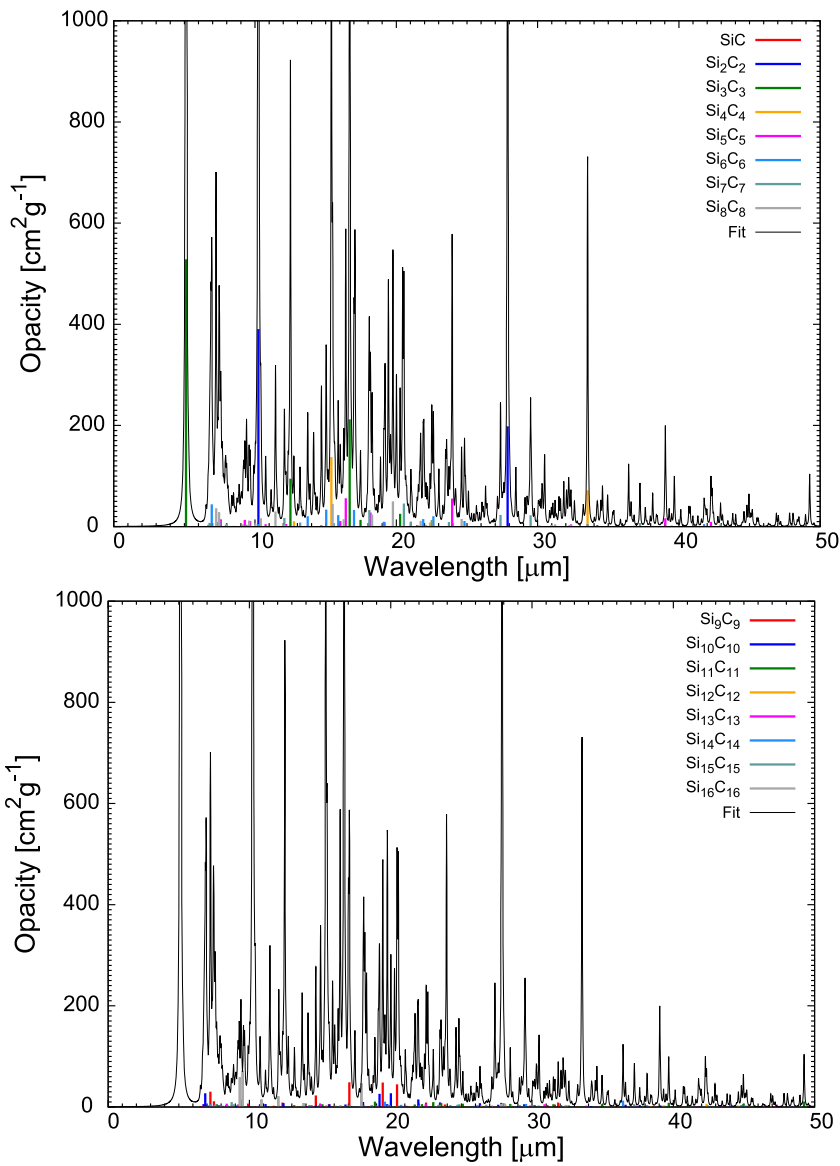

Figure 25. Vibrational spectra of the ground state $\mathrm{Si}_{n} \mathrm{C}_{n}$ clusters. The fit is a Lorentzian distribution with a FWHM parameter of 0.033 .

However, clusters with a symmetric arrangement show fewer emission modes, owing to the multiplicity of identical vibrations.

The unit conversion from $\mathrm{kmmole}^{-1}$ to the opacity in $\mathrm{cm}^{2} \mathrm{~g}^{-1}$ is obtained by

$$
1 \mathrm{~km} \mathrm{~mole}^{-1}=\frac{10^{5}}{\bar{\nu}} \mathrm{cm}^{2} \mathrm{~mole}^{-1}=\frac{10^{5}}{\bar{\nu} M} \mathrm{~cm}^{2} \mathrm{~g}^{-1},
$$


where $\bar{\nu}$ is the vibrational frequency in units of $\mathrm{cm}^{-1}$ and $M$ is the molecular mass in amu (e.g., $\sim 40$ for the $\mathrm{SiC}$ monomer).

\section{Discussion}

The $11.2-11.5 \mu \mathrm{m}$ feature observed in the spectra of C-rich stars represents the most prominent emission attributed to $\mathrm{SiC}$ dust particles. About $4 \%$ of the stars in the sample of LittleMarenin (1986) show a feature shifted to $11.6 \mu \mathrm{m}$ corresponding to the wavelength of the $\mathrm{SiC}$ molecule (monomer) vibration mode. Some $\mathrm{C}$ stars exhibit a second peak at around $11.7 \mu \mathrm{m}$ (Goebel et al. 1995). These stars are characterized by a lower feature strength and broadened emission indicating larger $\mathrm{SiC}$ particles. A spectral feature at $9 \mu \mathrm{m}$ that correlates with trends of the $11.3 \mu \mathrm{m}$ is observed as well in C stars (Speck et al. 2005). The authors conclude that the carrier of the $9 \mu \mathrm{m}$ is either an amorphous $\mathrm{SiC}$ or an Si-doped nanodiamond. Moreover, Speck et al. (2005) find that as the star evolves and increases its mass loss rate, the $\mathrm{SiC}$ dust grain sizes become smaller. Finally, in the superwind phase, owing to the high mass loss rate, the $\mathrm{SiC}$ feature appears in absorption, broadens, weakens, and is shifted toward shorter wavelengths. Speck et al. (2009) investigated spectral features in the $10-13 \mu \mathrm{m}$ range in a sample of extreme carbon stars and attributed them to carbonaceous solids including a fraction of $\mathrm{SiC}$ dust. In our study, $\mathrm{Si}_{2} \mathrm{C}_{2}$ at $10.237 \mu \mathrm{m}$ and $\mathrm{Si}_{3} \mathrm{C}_{3}$ at $12.507 \mu \mathrm{m}$ show the strongest emission in this range among the $\mathrm{Si}_{n} \mathrm{C}_{n}$ cluster ground states. Rau et al. (2015) showed that, apart from the spectral feature around $11.4 \mu \mathrm{m}$, amorphous carbon and $\mathrm{SiC}$ dust particles exhibit absorption distributions that are fairly similar in the small particle limit. This feature is thus a unique and distinct tracer for the presence of $\mathrm{SiC}$ dust grains. In addition, laboratory spectra show a wide variety of the $\mathrm{SiC}$ phonon features in the 10-13 $\mu \mathrm{m}$ wavelength range, in both peak wavelength and band shape (Mutschke et al. 1999). It is the only relatively broad band that is attributed to $\mathrm{SiC}$. As previously mentioned, the $\mathrm{SiC}$ crystal type ( $\alpha$ versus $\beta \mathrm{SiC}$ ) plays a minor role in the $10-13 \mu \mathrm{m}$ emissivity.

In our study, we found several clusters with vibrational emissions in the 11.2-11.5 $\mu \mathrm{m}$ wavelength. However, their overall IR intensity is too small to explain the observed emission. The investigated cluster sizes (up to $n=16$ ) may be too small to reproduce the bulk-related phonon emission around 11.3-11.4 $\mu \mathrm{m}$. The $\mathrm{SiC}$ molecule (monomer) exhibits a vibrational emission feature at $11.599 \mu \mathrm{m}$. However, its IR intensity is weak $\left(0.1274 \mathrm{~km} \mathrm{~mole}^{-1}=0.3694 \mathrm{~cm}^{2} \mathrm{~g}^{-1}\right)$ as compared with the IR intensities of the other $\mathrm{SiC}$ clusters. In addition, the three-atomic species $\mathrm{Si}_{2} \mathrm{C}$ and $\mathrm{SiC}_{2}$, the latter being a byproduct of $\mathrm{SiC}$ dust formation, cannot account for the $11.3 \mu \mathrm{m}$ feature in their spectra. $\mathrm{Si}_{3} \mathrm{C}_{3}$ (isomer $\mathrm{F}$ ) exhibits a feature at $11.325 \mu \mathrm{m}$ with a reasonable intensity of $106.2789 \mathrm{~km} \mathrm{~mole}^{-1}=100.3 \mathrm{~cm}^{2} \mathrm{~g}^{-1}$. However, the cluster is $0.84 \mathrm{eV}$ above the ground state at standard conditions, and the situation is similar $(0.8-1.2 \mathrm{eV})$ at characteristic wind conditions. $\mathrm{Si}_{4} \mathrm{C}_{4}$ (isomer $\mathrm{F}$ ) shows at $11.298 \mu \mathrm{m}$ a signature with a strength of $85.7498 \mathrm{~km} \mathrm{~mole}^{-1}=60.5501 \mathrm{~cm}^{2} \mathrm{~g}^{-1}$ and a potential energy of $0.4-0.6 \mathrm{eV}$ above the ground state, depending on the gas conditions. The ground state of $\mathrm{Si}_{5} \mathrm{C}_{5}$ (isomer A) shows an emission at $11.298 \mu \mathrm{m}$, but the IR intensity $\left(0.2336 \mathrm{~km} \mathrm{~mole}^{-1}\right)$ is (too) low. Other $\mathrm{Si}_{5} \mathrm{C}_{5}$ isomers show signatures in this wavelength range: structure $\mathrm{C}$ (which is the minimum structure in the higher-pressure cases) at $11.405 \mu \mathrm{m}$ with $3.1128 \mathrm{~km} \mathrm{~mole}^{-1}$, structure $\mathrm{D}$ at
Table 2

Vibrational Emission of the Presented Clusters $(n \geqslant 6)$ in the $11.2-11.5 \mu \mathrm{m}$ Wavelength Range

\begin{tabular}{|c|c|c|c|c|}
\hline$n$ & $\mathrm{X}$ & $\lambda($ in $\mu \mathrm{m})$ & $I$ (in $\mathrm{km} \mathrm{mole}^{-1}$ ) & $I\left(\right.$ in $\left.\mathrm{cm}^{2} \mathrm{~g}^{-1}\right)$ \\
\hline 6 & B & 11.365 & 1.0139 & 0.4801 \\
\hline 7 & B & 11.510 & 14.8496 & 6.1042 \\
\hline 8 & A & 11.449 & 83.6211 & 29.9181 \\
\hline 10 & A & 11.455 & 9.9048 & 2.8365 \\
\hline 11 & A & 11.384 & 8.1423 & 2.1066 \\
\hline 11 & D & 11.181 & 5.1542 & 1.3098 \\
\hline 12 & $\mathrm{C}$ & 11.424 & 3.9820 & 0.9477 \\
\hline 12 & D & 11.461 & 7.4771 & 1.7853 \\
\hline 13 & B & 11.449 & 3.4249 & 0.7541 \\
\hline 13 & $\mathrm{C}$ & 11.293 & 0.3679 & 0.080 \\
\hline 13 & D & 11.219 & 24.8402 & 4.9765 \\
\hline \multirow[t]{2}{*}{14} & $\mathrm{C}$ & 11.363 & 0.8570 & 0.1739 \\
\hline & & 11.289 & 2.8705 & 0.5787 \\
\hline 15 & B & 11.227 & 7.8250 & 1.4641 \\
\hline 15 & $\mathrm{C}$ & 11.213 & 4.8333 & 0.9032 \\
\hline 16 & $\mathrm{C}$ & 11.363 & 5.7596 & 1.0226 \\
\hline
\end{tabular}

Note. The first two columns identify cluster size $n$ and state X, the third column displays the wavelength $\lambda$ in $\mu \mathrm{m}$, and the fourth column lists intensity $I$ (in $\mathrm{km} \mathrm{mole}^{-1}$ and $\mathrm{cm}^{2} \mathrm{~g}^{-1}$ ).

$11.424 \mu \mathrm{m}$ and $3.3497 \mathrm{~km} \mathrm{~mole}^{-1}$, structure $\mathrm{E}$ at $11.350 \mu \mathrm{m}$ with $0.5734 \mathrm{~km} \mathrm{~mole}^{-1}$, and structure $\mathrm{F}$ at $11.299 \mu \mathrm{m}$ with $3.5731 \mathrm{~km} \mathrm{~mole}^{-1}$. Although $\mathrm{Si}_{5} \mathrm{C}_{5}$ has several low-lying candidate carriers of $11.3 \mu \mathrm{m}$ features, the IR intensities are very low compared with the other spectral features these isomers have. For the larger clusters, we compiled a table with vibrational intensities in the 11.2-11.5 $\mu \mathrm{m}$ range (see Table 2).

For cluster sizes of $n=8,11,12,13$, and 15, the ground states (or next-higher-lying states) emit in this wavelength regime; however, the intensities are (apart from 8A) quite low. Large abundances of a specific cluster, though, could increase the intensity significantly.

Some of the spectral peaks identified in our calculations are not explicitly reported in the literature. Nevertheless, SiC clusters may represent a key player for the onset of dust formation in C-rich AGB stars, albeit not directly detected.

In fact, Frenklach et al. (1989) found experimental evidence for a scenario in which $\mathrm{SiC}$ nucleates at higher temperatures and provides surfaces for subsequent carbon condensation in a hydrogen-rich atmosphere. Moreover, Cadwell et al. (1994) used the model of induced nucleation, where the grain growth proceeds on reactive surfaces of pre-existing seed particles, and showed that a subsequent condensation of carbonaceous material results in composite grains that are consistent with grains found in pristine meteorites. Kozasa et al. (1996) thus suggested that $\mathrm{SiC}$ grains form at high temperatures by homogeneous nucleation but that as soon as the temperature has decreased (i.e., at larger radii), a mantle of amorphous carbon $(\mathrm{amc}$ ) may deposit on $\mathrm{SiC}$ seeds. The spectral signatures of pure $\mathrm{SiC}$ may thus be blended and/or suppressed by the amc mantle.

There is a series of unidentified bands (UIBs) at 3.3, 6.2, 7.7, 8.6, and $12.7 \mu \mathrm{m}$, respectively, seen in carbon-rich AGB stars. These features are commonly attributed to polycyclic aromatic hydrocarbon (PAH) emission (Beintema et al. 1996; Hron et al. 1998; Jørgensen et al. 2000; Boersma et al. 2006). As both PAHs and $\mathrm{SiC}$ clusters contain aromatic $\mathrm{C}_{6}$ rings and have conjugated bonds in common, they may show a remarkable 
spectral similarity. In the following text, we examine whether or not $\mathrm{SiC}$ clusters could account for the emission of UIBs. For the 3.3 and $6.2 \mu \mathrm{m}$ band we find no coincidence with the vibrational spectra of $\mathrm{SiC}$ clusters. Around $7.7 \mu \mathrm{m}$ the $\mathrm{SiC}$ ground state clusters with $n=11,13,14$, and 16 show emission. We find that the ground states $\mathrm{Si}_{15} \mathrm{C}_{15}$ and $\mathrm{Si}_{16} \mathrm{C}_{16}$ emit at $8.6 \mu \mathrm{m}$. Around $12.7 \mu \mathrm{m}$ we find vibrational modes of the $n=14$ and $n=15$ ground states. All these structures have $\mathrm{C}_{6}$ and $\mathrm{C}_{5}$ rings in common. The symmetric "bucky"-like structure B in Figure 16 and structure A in Figure 19 do not exhibit spectral features at these wavelengths.

Next, we address the viability of SiC cluster nucleation and the derivation of (parametrized) reaction rates from the analysis of our results. So far, we identify the most likely cluster structures and pathways in $\mathrm{SiC}$ nucleation and dust formation. However, rate determination is difficult to achieve, as it requires knowledge about the (various) transition states involved in the reaction. A directly proceeding reaction could be evaluated, as it depends only on the (calculated) properties of reactants and the products. Such reactions are, however, unlikely to occur, in particular for the gas-phase chemical reactions of $\mathrm{SiC}$ monomers and dimers representing the crucial starting point in the present bottom-up approach. Unfortunately, these rates are poorly characterized. Only two chemical reaction rates for the $\mathrm{SiC}$ monomer are reported in NIST (Linstrom \& Mallard 2005):

1. $\mathrm{Si}+\mathrm{C} \rightarrow \mathrm{SiC}+\mathrm{h} \nu$ (radiative association)

2. $\mathrm{Si}+\mathrm{CH}_{2} \rightarrow \mathrm{SiC}+\mathrm{CH}$ (bimolecular collision)

whereas the bimolecular collision reaction is estimated by analogy to the reaction $\mathrm{Si}+\mathrm{CH}_{3} \rightarrow \mathrm{SiCH}+\mathrm{H}_{2}$ (Kunz \& Roth 2001). Despite its low energy barrier of $136.73 \mathrm{~K}$ $\left(1.14 \mathrm{~kJ} \mathrm{~mol}^{-1}\right)$ the radiative association reaction is very slow (Andreazza et al. 2009). Moreover, owing to the lack of gas phase reaction rates, isovalences of $\mathrm{Si}$ and $\mathrm{C}$ are presumed and rates for $\mathrm{SiC}$ are equalized with rates for $\mathrm{C}_{2}$ (see e.g., Cherchneff \& Dwek 2010). This may be adequate as a first approximation. However, the binding energy of $\mathrm{SiC}(4.71 \mathrm{eV})$ is higher by more than $1 \mathrm{eV}$ compared with $\mathrm{C}_{2}(3.6 \mathrm{ev})$. Moreover, the $\mathrm{Si}-\mathrm{C}$ bonding has a small but not negligible dipole moment of $\sim(1.7-1.8)$ Debye, due to the larger size and the higher number of electrons of the $\mathrm{Si}$ atom. This may have non-negligible effects on the reactivity of the molecules.

Yasuda \& Kozasa (2012) provide reaction enthalpies for $\mathrm{SiC}$ cluster growth for temperatures of 1500 and $1000 \mathrm{~K}$. The enthalpies indicate that a homomolecular cluster growth (i.e., the addition of $\mathrm{SiC}$ molecules to an $\mathrm{Si}_{n} \mathrm{C}_{n}$ cluster) is the energetically most favorable formation route. Albeit that the reaction enthalpies are approximated with that of solid $\mathrm{SiC}$ for $\mathrm{n}>3$, they concluded that the reactions

$$
\mathrm{Si}_{n} C_{n}+\mathrm{SiC} \rightarrow \mathrm{Si}_{n+1} \mathrm{C}_{n+1}
$$

are the dominant processes in the formation of $\mathrm{SiC}$ dust grains, consistent with our findings (see Figure 22).

In the following, we list observations of $\mathrm{Si}-\mathrm{C}$ molecules in C-rich AGB stars and compare them with our findings. The molecular $\mathrm{SiC}$ radical has been detected first in $\mathrm{CW}$ Leo by Cernicharo et al. (1989). We find a rotational constant of $20643.1 \mathrm{MHz}$ consistent with the spectroscopic constant $B=20297.6 \mathrm{MHz}$. Note that $\mathrm{SiC}$ is a triplet and thus the rotational level is split into three states. Our M11 calculations of $\mathrm{Si}_{2} \mathrm{C}$ yield the following rotational constants $A=58363.8 \mathrm{MHz}$,
$B=4567.1 \mathrm{MHz}$, and $C=4235.7 \mathrm{MHz}$, whereas the derived constants in Cernicharo et al. (2015) (S reduction) as $A=64074.3$, $B=4395.5$, and $C=4102.1$ are slightly different but still compatible. For $\mathrm{SiC}_{2}$, we obtain the following rotational constants $A=53511.7 \mathrm{MHz}, B=13004.6 \mathrm{MHz}$, and $C=10462.1$, which are in good agreement with the laboratory $(A=53909 \mathrm{MHz}$, $B=13530 \mathrm{MHz}, \quad C=10751 \mathrm{MHz})$ and observational data $(A=52390 \mathrm{MHz}, B=13156.2 \mathrm{MHz}, C=10447.4 \mathrm{MHz})$ of Thaddeus et al. (1984). The most stable isomer of $\mathrm{SiC}_{3}$ has a cyclic geometry and was detected in CW Leo (Apponi et al. 1999; Cernicharo et al. 2000). We find rotational constants of 39.962 and $6.240 \mathrm{GHz}$ with the M11 functional, which is relatively close to the laboratory spectra of 37.9 and $5.83 \mathrm{GHz}$, respectively. Linear $\mathrm{SiC}_{4}$ was detected by Ohishi et al. (1989) in CW Leo. With the M11 functional we obtain a rotational constant of $1549.6 \mathrm{MHz}$, close to the observed value of $1533.8 \mathrm{MHz}$.

Recent observations revealed that among the $\mathrm{SiC}$ molecules, $\mathrm{SiC}_{2}$ and $\mathrm{Si}_{2} \mathrm{C}$ dominate the inner envelope, whereas the $\mathrm{SiC}$ molecule is two to three orders of magnitude less abundant (Cernicharo et al. 2015). We conclude that the SiC molecule is rapidly converted in the $\mathrm{SiC}_{2}, \mathrm{Si}_{2} \mathrm{C}$, and $\mathrm{Si}_{n} \mathrm{C}_{n}$ clusters. The emission of $\mathrm{SiC}_{3}$ and $\mathrm{SiC}_{4}$ arises in the intermediate and outer envelope of $\mathrm{CW}$ Leo. Thus, we suggest that the latter molecules are the result of photochemistry or grain surface reactions and that they do not play a role in the nucleation of $\mathrm{SiC}$ dust. Assuming a dust-to-gas mass ratio of $2.5 \times 10^{-3}$ and a fraction of $10 \% \mathrm{SiC}$ in the dust grains results in a solid $\mathrm{SiC}$ abundance, $(\mathrm{SiC})_{\text {dust }} / \mathrm{H}_{2}$, of $1.25 \times 10^{-5}$. This is slightly less than half of the solar $\mathrm{Si}$ abundance $\left(3 \times 10^{-5}\right)$. It has been suggested that the recently discovered $\mathrm{Si}_{2} \mathrm{C}$ molecule plays a key role in the formation of $\mathrm{SiC}$ dust grains (Cernicharo et al. 2015). Although this molecule is well characterized in terms of geometry and energetics, reaction rates are lacking for $\mathrm{Si}_{2} \mathrm{C}$. In our study, we find that the Gibbs free energy of formation of $\mathrm{SiC}_{2}$ is lower by at least $100 \mathrm{~kJ} \mathrm{~mol}^{-1}$ compared to that of $\mathrm{Si}_{2} \mathrm{C}$ for all $p-T$ combinations listed in Table 1 . In fact, the latter explains the observed higher $\mathrm{SiC}_{2}$ abundance between 1 and $4 R_{*}$ compared with $\mathrm{Si}_{2} \mathrm{C}$. In the intermediate envelope region (4-40 $R_{*}$ ), equal amounts of $\mathrm{Si}_{2} \mathrm{C}$ and $\mathrm{SiC}_{2}$ are present. Further out ( $\sim 40-1000 R_{*}$ ), again $\mathrm{SiC}_{2}$ is favored over $\mathrm{Si}_{2} \mathrm{C}$, before both species are essentially dissociated/depleted. These results indicate that $\mathrm{SiC}_{2}$ is favored over (or is at least equivalent to) $\mathrm{Si}_{2} \mathrm{C}$ in circumstellar outflows and agree with our calculations, assuming a formation pathway via the $\mathrm{SiC}$ molecule and equal amounts of $\mathrm{Si}$ and $\mathrm{C}$ atoms. As $\mathrm{C}$ is $\sim 17$ times more abundant than $\mathrm{Si}$, assuming scaled-solar abundances, the dominance of $\mathrm{SiC}_{2}$ versus $\mathrm{SiC}$ and $\mathrm{Si}_{2} \mathrm{C}$ is even emphasized. Owing to the excess of $\mathrm{C}$ relative to $\mathrm{Si}$, the molecular species $\mathrm{Si}_{3} \mathrm{C}$ and $\mathrm{Si}_{4} \mathrm{C}$ are excluded from the present study. In the C-rich atmospheres of evolved AGB stars, this $(\mathrm{C} / \mathrm{Si}$ ) ratio tends to be even higher and has values $\sim 20-30$ (Cristallo et al. 2015). As can be evaluated from Table 3, cluster growth via $\mathrm{SiC}_{2}$ is energetically unfavorable at temperatures of $1000 \mathrm{~K}$ and $1500 \mathrm{~K}$. The formation of $\mathrm{SiC}_{2}$ thus represents a competing branching to the synthesis of $\mathrm{Si}_{n} \mathrm{C}_{n}, n \geqslant 3$ clusters for conditions close to the star. Therefore, we conclude that $\mathrm{SiC}_{2}$ is a byproduct of $\mathrm{SiC}$ dust formation in the inner envelope. However, farther from the star, at lower temperatures and pressures, the cluster nucleation via $\mathrm{SiC}_{2}$ becomes exothermic and exergonic and is thus also likely to occur. A nucleation pathway involving $\mathrm{Si}_{2} \mathrm{C}$ as an intermediary is energetically thoroughly viable and close to the star, 
Table 3

Reaction Enthalpy $\Delta \mathrm{H}^{0}$ in $\mathrm{kJ}$ mole ${ }^{-1}$ for SiC Cluster Growth Reactions and Comparison with the Studies of Yasuda \& Kozasa (2012) and Deng et al. (2008) at Temperatures of $1000 \mathrm{~K}$ and $1500 \mathrm{~K}$

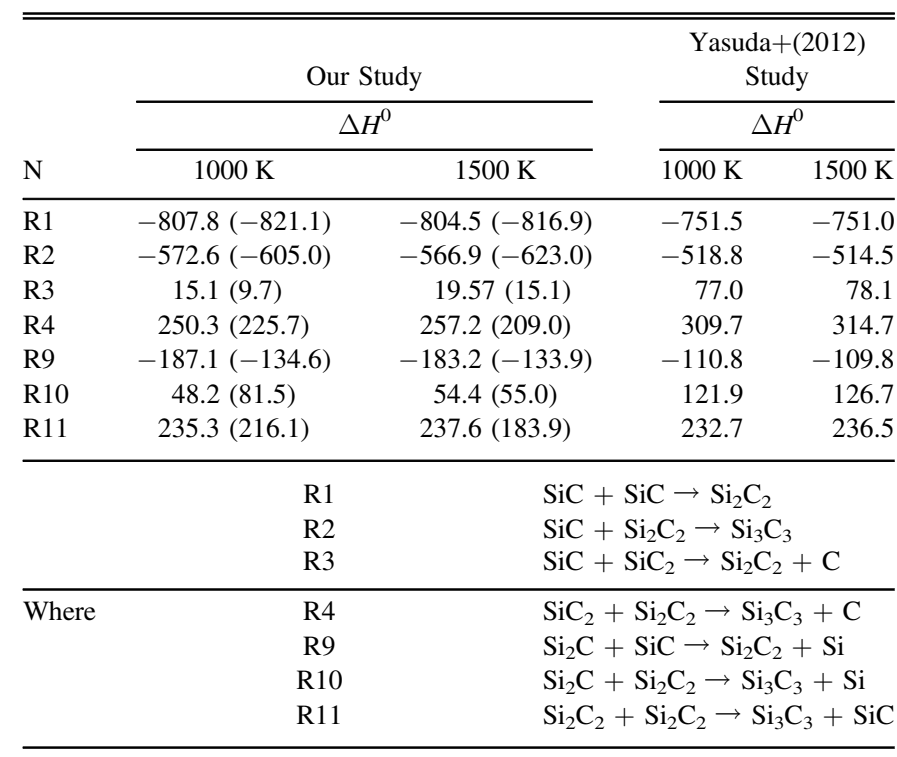

although a cluster growth according to Equation (6) is expected to be faster and more efficient. Our theoretical findings thus explain and reflect the observed radial abundance profiles of the $\mathrm{Si}-\mathrm{C}$ molecules in $\mathrm{CW}$ Leo.

In Table 3 we compare the energetics of the ground-state clusters derived in this study with Yasuda \& Kozasa (2012). The authors evaluated the reaction enthalpies $\Delta H^{0}$ from a data set by Deng et al. (2008) at $1000 \mathrm{~K}$ and $1500 \mathrm{~K}$. We find similar trends in the exothermicity of the reactions, though our values are systematically lower by $3-77(16-86) \mathrm{kJ} \mathrm{mol}^{-1}$ for $T=1000 \mathrm{~K}$ and by $24-108 \mathrm{~kJ} \mathrm{~mol}^{-1}$ for $T=1500 \mathrm{~K}$.

Further listed reactions in Yasuda \& Kozasa (2012) could not be compared as we have not investigated the species $\mathrm{SiC}_{3}$, $\mathrm{SiC}_{4}, \mathrm{Si}_{2} \mathrm{C}_{3}, \mathrm{Si}_{3} \mathrm{C}, \mathrm{Si}_{3} \mathrm{C}_{2}, \mathrm{Si}_{4} \mathrm{C}, \mathrm{Si}_{4} \mathrm{C}_{2}$, and $\mathrm{Si}_{5} \mathrm{C}$. The reason for the systematic offset may arise due to the use of different functionals/basis sets in Deng et al. (2008) (M11/cc-pVTZ and B3LYP/cc-pVTZ versus B3PW91/6-31G(d)), other ground state clusters, and the use of combined thermochemistry databases.

Another point to address is the cluster physics. Therefore, we compare our results to studies of silicon oxide ( $\mathrm{SiO}$ ) representing a counterpart to $\mathrm{SiC}$ in O-rich environmentsand titanium carbide (TiC), another metallic carbide.

$\mathrm{SiO}$ is a key ingredient for the formation of the astronomically relevant and abundant silicates of pyroxene and olivine. Under circumstellar conditions, homogeneous $\mathrm{SiO}$ nucleation is limited by considerably large energy barriers of the order $\sim 1 \mathrm{eV}$ (Goumans \& Bromley 2012; Bromley et al. 2016). Moreover, the $(\mathrm{SiO})_{n}$ global minimum structures show segregations in the form of $\mathrm{Si}-\mathrm{Si}$ bonds for sizes $n>5$. In contrast to $\mathrm{SiO}$ clusters, $(\mathrm{SiO})_{n}$, the most energetically favorable $\mathrm{SiC}$ clusters tend to have alternating $\mathrm{Si}-\mathrm{C}$ bonds for sizes larger than 12 units and exhibit segregations for sizes $n<12$. We thus observe opposing trends in the degree of segregation versus size for $\mathrm{SiO}$ and $\mathrm{SiC}$ clusters. Furthermore, homogeneous $\mathrm{SiC}$ nucleation is feasible in cirumstellar environments and may occur even at elevated temperatures $(T=2000 \mathrm{~K})$. TiC is found in the centers of pristine meteoric grains, and laboratory measurements of small-sized TiC nanocrystals show a prominent spectral feature of around $21 \mu \mathrm{m}$ (von Helden et al. 2000). However, Chigai et al. (2003) demonstrated that $\mathrm{TiC}$ grains are implausible carriers of the observed IR $21 \mu \mathrm{m}$ feature around C-rich post-AGB stars. Recent investigations of small $(\mathrm{TiC})_{n}(n=6,12)$ clusters have shown that the lowest-energy structures possess a cubic geometry with alternating Ti-C bondings (Lamiel-Garcia et al. 2012). Isomers deviating from pure alternating bonds (i.e., exhibting $\mathrm{C}-\mathrm{C}$ bonds) have potential energies that are slightly above the cubic forms. We thus conclude that segregation plays a negligible (or minor) role in homogeneous TiC nucleation and that the transition to the crystalline bulk material takes place at comparably small sizes.

\section{Summary}

We have found energetically favorable clusters for $(\mathrm{SiC})_{n}$ up to a size of $n=16$. The results are used to predict the viability of nucleation and the reaction probability in $\mathrm{SiC}$ cluster chemistry. Our findings show that $\mathrm{SiC}$ dust formation is viable in the dense cooling atmospheric gas layers by the addition of single $\mathrm{SiC}$ gas-phase molecules (homogeneous nucleation). The nucleation pathway includes waiting points, where the $\mathrm{SiC}$ addition may be energetically unfavorable. Nevertheless, nucleation owing to changes in gas conditions (e.g., shocks, radiation) is not unlikely. The $11.3 \mu \mathrm{m}$ feature represents an emission that is uniquely attributable to $\mathrm{SiC}$ dust grains in the near-IR regime. There are a number of clusters showing emission around this feature. However, their overall intensities are rather low. We thus conclude that the major contribution to $11.3 \mu \mathrm{m}$ emission arises from bulk $\mathrm{SiC}$ material. The rate enthalpies compare qualitatively well with a previous study (Yasuda \& Kozasa 2012), although the data derived by our study is systematically lower by $\sim 3-77(16-86) \mathrm{kJ} \mathrm{mol}^{-1}$.

We acknowledge the CINECA award under the ISCRA initiative for the availability of high-performance computing resources and support. The authors gratefully acknowledge the referee whose constructive comments greatly improved the quality of the present paper. We acknowledge L. Decin for useful discussions on the comparisons with observations. The figures displaying cluster structures have been produced with the help of the program MOLDEN (Schaftenaar \& Noordik 2000). All other figures have been created with gnuplot.

\section{References}

Al-Sunaidi, A. A., Sokol, A. A., Catlow, C. R. A., \& Woodley, S. M. 2008, The Journal of Physical Chemistry C, 112, 18860

Amari, S., Lewis, R. S., \& Anders, E. 1994, GeCoA, 58, 459

Amari, S., Zinner, E., \& Gallino, R. 2014, in AIP Conf. Ser. 1594, 12th International Symposium on Origin of Matter and Evolution of Galaxies, ed. S. Jeong et al. (Melville, NY: AIP), 307

Andreazza, C. M., Vichietti, R. M., \& Marinho, E. P. 2009, MNRAS, 400, 1892

Apponi, A. J., McCarthy, M. C., Gottlieb, C. A., \& Thaddeus, P. 1999, ApJL, 516, L103

Becke, A. D. 1993, JChPh, 98, 1372

Beintema, D. A., van den Ancker, M. E., Molster, F. J., et al. 1996, A\&A, 315, L369

Belenkov, E. A., Agalyamova, E. N., \& Greshnyakov, V. A. 2012, PhSS, 54, 433

Bernatowicz, T., Fraundorf, G., Ming, T., et al. 1987, Natur, 330, 728 
Bernatowicz, T. J., Akande, O. W., Croat, T. K., \& Cowsik, R. 2005, ApJ, 631, 988

Bertolus, M., Finocchi, F., \& Millie, P. 2004, JChPh, 120, 4333

Boersma, C., Hony, S., \& Tielens, A. G. G. M. 2006, A\&A, 447, 213

Brenner, D. W. 1990, PhRvB, 42, 9458

Bromley, S. T., Gomez Martin, J. C., \& Plane, J. M. C. 2016, PCCP, 18, 26913

Byrd, J. N., Lutz, J. J., Jin, Y., et al. 2016, JChPh, 145, 024312

Cadwell, B. J., Wang, H., Feigelson, E. D., \& Frenklach, M. 1994, ApJ, 429,285

Cernicharo, J., Gottlieb, C. A., Guelin, M., Thaddeus, P., \& Vrtilek, J. M. 1989, ApJL, 341, L25

Cernicharo, J., Guélin, M., \& Kahane, C. 2000, A\&AS, 142, 181

Cernicharo, J., McCarthy, M. C., Gottlieb, C. A., et al. 2015, ApJL, 806, L3

Cherchneff, I., \& Dwek, E. 2010, ApJ, 713, 1

Chigai, T., Yamamoto, T., Kaito, C., \& Kimura, Y. 2003, ApJ, 587, 771

Cristallo, S., Straniero, O., Piersanti, L., \& Gobrecht, D. 2015, ApJS, 219, 40

Daulton, T. L., Bernatowicz, T. J., Lewis, R. S., et al. 2003, GeCoA, 67, 4743

Davis, A. M. 2011, PNAS, 108, 19142

Demiroglu, I., Tosoni, S., Illas, F., \& Bromley, S. T. 2014, Nanos, 6, 1181

Deng, J. L., Su, K. H., Wang, X., et al. 2008, EPJD, 49, 21

Dorfi, E. A., \& Hoefner, S. 1991, A\&A, 248, 105

Draine, B. T. 2009, in in ASP Conf. Ser. 414, Cosmic Dust-Near and Far, ed. T. Henning, E. Grün, \& J. Steinacker (San Francisco, CA: ASP), 453

Duan, X. F., Burggraf, L. W., \& Huang, L. 2013, Molecules, 18, 8591

Duan, X., Wei, J., Burggraf, L., \& Weeks, D. 2010, ComMS, 47, 630

Ehrenfreund, P., \& Charnley, S. B. 2000, ARA\&A, 38, 427

Erhart, P., \& Albe, K. 2005, PhRvB, 71, 035211

Ferrarotti, A. S., \& Gail, H.-P. 2006, A\&A, 447, 553

Frenklach, M., Carmer, C. S., \& Feigelson, E. D. 1989, Natur, 339, 196

Gale, J. D. 1997, J. Chem. Soc., Faraday Trans., 93, 629

Gallino, R., Raiteri, C. M., Busso, M., \& Matteucci, F. 1994, ApJ, 430, 858

Gobrecht, D., Cherchneff, I., Sarangi, A., Plane, J. M. C., \& Bromley, S. T. 2016, A\&A, 585, A6

Goebel, J. H., Cheeseman, P., \& Gerbault, F. 1995, ApJ, 449, 246

Goumans, T. P. M., \& Bromley, S. T. 2012, MNRAS, 420, 3344

Henning, T. (ed.) 2010, Astromineralogy (Berlin: Springer)

Höfner, S. 2008, A\&A, 491, L1

Hoppe, P., Leitner, J., Gröner, E., et al. 2010, ApJ, 719, 1370

Hoppe, P., Strebel, R., Eberhardt, P., Amari, S., \& Lewis, R. S. 1996, GeCoA, 60,883

Hou, J., \& Song, B. 2008, JChPh, 128, 154304

Hron, J., Loidl, R., Hoefner, S., et al. 1998, A\&A, 335, L69

Hynes, K. M., \& Gyngard, F. 2009, in Lunar and Planetary Science Conf. 40, 1198

Jørgensen, U. G., Hron, J., \& Loidl, R. 2000, A\&A, 356, 253

Koehler, T. M., Gail, H.-P., \& Sedlmayr, E. 1997, A\&A, 320, 553

Kozasa, T., Dorschner, J., Henning, T., \& Stognienko, R. 1996, A\&A, 307,551

Kunz, A., \& Roth, P. 2001, International Journal of Chemical Kinetics, 33, 741
Lamiel-Garcia, O., Bromley, S. T., \& Illas, F. 2012, ESPA, 5, 213

Lee, G., Helling, C., Giles, H., \& Bromley, S. T. 2015, A\&A, 575, A11

Linstrom, P. J., \& Mallard, W. G. (ed.) 2005, NIST Chemistry WebBook, NIST Standard Reference Database Number 69 (Gaithersburg, MD: National Institute of Standards and Technology)

Little-Marenin, I. R. 1986, ApJL, 307, L15

Liu, N., Savina, M. R., Davis, A. M., et al. 2014, ApJ, 786, 66

Liu, N., Savina, M. R., Gallino, R., et al. 2015, ApJ, 803, 12

McCarthy, M. C., Baraban, J. H., Changala, P. B., et al. 2015, The Journal of Physical Chemistry Letters, 6, 2107

McQuarrie, D., \& Simon, J. 1999, Molecular Thermodynamics (Mill Valley, CA: Univ. Science Books)

Mühlhäuser, M., Froudakis, G., Zdetsis, A., et al. 1994, ZPhyD, 32, 113

Mutschke, H., Andersen, A. C., Clément, D., Henning, T., \& Peiter, G. 1999, A\&A, 345, 187

Ohishi, M., Kaifu, N., Kawaguchi, K., et al. 1989, ApJL, 345, L83

Patnaik, P. 2003, Handbook of Inorganic Chemicals (New York: McGraw-Hill)

Peverati, R., \& Truhlar, D. G. 2012, The Journal of Physical Chemistry Letters, 3, 117

Pradhan, P., \& Ray, A. K. 2004, arXiv:physics/0408016

Rau, G., Paladini, C., Hron, J., et al. 2015, A\&A, 583, A106

Reilly, N. J., Changala, P. B., Baraban, J. H., et al. 2015, JChPh, 142, 231101

Schaftenaar, G., \& Noordik, J. 2000, JCAMD, 14, 123

Song, B., Yong, Y., Hou, J., \& He, P. 2010, EPJD, 59, 399

Speck, A. K., Barlow, M. J., \& Skinner, C. J. 1996, NASCP, 3343, 61

Speck, A. K., Cami, J., Markwick-Kemper, C., et al. 2006, ApJ, 650, 892

Speck, A. K., Corman, A. B., Wakeman, K., Wheeler, C. H., \& Thompson, G. 2009, ApJ, 691, 1202

Speck, A. K., Thompson, G. D., \& Hofmeister, A. M. 2005, ApJ, 634, 426

Stillinger, F. H., \& Weber, T. A. 1985, PhRvB, 31, 5262

Stroud, R. M., \& Bernatowicz, T. J. 2005, LPI, 36, 2010

Tersoff, J. 1989, PhRvB, 39, 5566

Testi, L., Birnstiel, T., Ricci, L., et al. 2014, in Protostars and Planets VI, ed. H. Beuther et al. (Tucson, AZ: Univ. of Arizona Press), 339

Thaddeus, P., Cummins, S. E., \& Linke, R. A. 1984, ApJL, 283, L45

van Duin, A., Dasgupta, F., Lorant, F., \& Goddard, W. 2001, JPCA, 105, 9396 Vashishta, P., Kalia, R. K., Nakano, A., \& Rino, J. P. 2007, JAP, 101, 103515 von Helden, G., Tielens, A. G. G. M., van Heijnsbergen, D., et al. 2000, Sci, 288,313

Wales, D. J., \& Doye, J. P. K. 1997, JPCA, 101, 5111

Watkins, M. B., Shevlin, S. A., Sokol, A. A., et al. 2009, PCCP, 11, 3186

Whitmore, L., Sokol, A. A., \& Catlow, C. R. A. 2002, SurSc, 498, 135

Woitke, P. 2006, A\&A, 460, L9

Woitke, P., Helling, C., Winters, J. M., \& Jeong, K. S. 1999, A\&A, 348, L17

Wright, K., \& Jackson, R. A. 1995, J. Mater. Chem., 5, 2037

Yasuda, Y., \& Kozasa, T. 2012, ApJ, 745, 159

Zhukovska, S., \& Henning, T. 2013, A\&A, 555, A99

Zinner, E., Amari, S., Guinness, R., et al. 2007, GeCoA, 71, 4786 